\title{
Unsteady Couette Flow Past Between Two HorizontalRiga Plates With Hall and Ion Slip Current
}

Sonia Nasrin ( $\nabla$ sn_mri@yahoo.com )

Jagannath University

\section{Rabindra Nath Mondal}

Jagannath University

Md. Mahmud Alam

Khulna University

\section{Research Article}

Keywords: Couette Flow, Riga plate, Hall and Ion-Slip Current, Explicit Finite difference

Posted Date: June 4th, 2021

DOI: https://doi.org/10.21203/rs.3.rs-574292/v1

License: (1) This work is licensed under a Creative Commons Attribution 4.0 International License.

Read Full License 


\title{
Unsteady Couette Flow past Between Two Horizontal Riga Plates with Hall and Ion Slip Current
}

\author{
S. Nasrin ${ }^{1}$, R. N. Mondal ${ }^{1}$ and M. M. Alam ${ }^{2}$ \\ ${ }^{1}$ Department of Mathematics, Jagannath University, Dhaka-1100, Bangladesh \\ ${ }^{2}$ Mathematics Discipline, Khulna University, Khulna-9208, Bangladesh \\ Email: sn_mri@yahoo.com
}

\begin{abstract}
In this article, we have investigated an unsteady Couette flow past between two horizontal Riga plates with the Hall and Ion-slip effects.Riga plate is the combination of electrodes and permanent magnets that creates a plane surface which produced the electromagnetic hydrodynamic fluid behavior and mostly uses in industrial processes with fluid flow affairs. It is used as an agent of reduce the skin friction and also diminish the turbulent effects. The numerical solutions are acquired by using explicit finite difference method and estimated results have been gained for various values of dimensionless pressure gradient parameter, the Hall and Ion-slip parameters, modified Hartmann number, Prandtl number, Eckert number. The expression of skin friction and Nusselt number has been computed and the outcomes of the relevant parameters on various distributions have been sketched and presented as well as graphically.
\end{abstract}

Keywords: Couette Flow; Riga plate; Hall and Ion-Slip Current; Explicit Finite difference

\section{Introduction}

The highly electrically conducting fluid is controlled by magneto hydrodynamics flow with the influence of external magnetic field is known as classical MHD flow control .When the fluid is too weak in electrically conducting so that the Lorentz force decreases exponentially that's why an external electric field must be applied to achieve efficient flow control, this is called Electro-magneto hydrodynamic (EMHD) flow. There are tremendoususe of poor conductivity fluids in the field of magneto-aerodynamics, civil engineering, mechanical engineering, chemical engineering, dust or fumes in a gas, in biomechanics and purification the groundwater and oil. The combination of electrodes and permanent magnets creates a plane surface instead of polarity and magnetization is Riga plate that is introduced by Gailitis and Leilausis [1].This order minimizes the friction and pressure drag of submarines. It is also restrained the separation of boundary layer as well as diminishing the turbulence effects. When the flow passes, the Lorentz force is created by gather the electrodes and permanent magnets in the flat surface. A turbulent channel event with small Reynolds number is utilized the Lorentz force discovered by Berger et al. [2]. Pantokratoras and Magyari [3] proposed the electro-magneto hydrodynamic free convection fluid flow with a poor conductivity along a Riga plate. Wahidunnisa et al. [4] studied the heat source of a nanofluid flow through a Riga plate with viscous dissipation. Iqbal et al.[5] explored their idea on an electrically conducted Riga plate with viscous dissipation and thermal radiation of nano fluid with melting heat and they use the Keller Box scheme to obtain the solution. Ayub et al. [6] examined the effect of EMHD nano fluid flow along an electromagnetic actuator. Ahmed et al. [7] carried out the mixed convection of a nano fluid flow along a vertical Riga plate with the effect of a strong suction. The physical problems of magneto-hydrodynamic flows with Hall and Ion-slip current have its practical applications as electromagnetic flow meters, electromagnetic pumps and MHD power generator, aerodynamic heating, electrostatic precipitation, geophysics, astrophysics and many engineering and industrial processes [8]. Javeri[9] investigated the combined effect of Hall and Ion slip currents, Joule heating and viscous dissipation on the laminar MHD channel with heat transfer. Eraslan[10] expressed a distribution of temperature for the MHD channels with Hall effect. The MHD laminar flow along a porous medium has significant applications in engineering and agricultural process, groundwater flows, petroleum industry, and oil and gas purification. A lot of research work has been held on the MHD steady or unsteady flows over a vertical porous plate with Hall and ion-slip under different physical effects has been studied of their wide applications. From the point of view, the effect of Hall current and ion-slip with heat and mass convection flows of an electrically conducting fluid has been discussed by several authors such as Seddeek and Aboeldahab [11] are investigated an unsteady free convection and electrically conducting flow with the Hall currents and radiation effect of gray gas along an infinite vertical porous plate where a strong transverse magnetic field is imposed perpendicularly to the plate. Narayana et al. [12] examined the heat and mass transfer with Hall current along a vertical porous plate under the combination of buoyancy force of thermal and species diffusion in the presence of a transversely applied uniform magnetic field. Debnath et al. [13] studied the effects of Hall current on unsteady hydro magnetic flow past a porous plate in a rotating fluid system. Nimr and Masoud [14] discussed an unsteady free convection flow in a porous media along a flat plate. Krishna et al. [15] carried out an unsteady free convective magneto- 
hydrodynamic flow with Hall and ion slip current effects through an accelerated inclined plate with rotation which is surrounded by a porous medium with the effect of inclined angle also with the change of reference frame. They have used Laplace transform to solve these problems analytically.Sharma et al. [16]considered a viscous, unsteady and incompressible fluid flow along a vertically infinite porous plate with Hall effect and heat source (or sink). Angirasa and Peterson [17] presented a numerical study on heat transfer in natural transmission from an isothermal vertical surface which is a stable layered to a fluid-saturated thermally stratified porous medium. Kumar and Singh [18] analyzed the heat transfer from a vertically isothermal surface with the impact of thermal stratification in a porous medium. The influence of MHD Couette flows with Hall and Ion slip current has a great importance of experimental and theoretical applications in magnetic material processing, astrophysics, polymer technology, heating electrostatics, nuclear engineering, pumps and power generators, geophysical and industrial fields. These problems have been investigated by the authors such as Ghara et al. [19] studied an electrically conducting fluid of an unsteady Couette flow between two horizontal porous plates with Hall and Ion-slip effects. An unsteady Couette flow of an incompressible fluid with Ion-slip effect and an external uniform magnetic field is applied perpendicular to the plates isexamined in [20] and the same flow with uniform suction and injection is bounded by two parallel porous plates with heat transfer is discussed in [21] by Attia. Kumar et al. [22] discussed the Couette flow in three dimensional with heat transfer through a porous medium bounded by infinite vertical porous plates. From the great interest in unsteady laminar Couette flow of its great applications in environmental, industrial, biomedical, engineering, nuclear reactors and oil purifications, many researchers [23,24,25]have expressed their views on its.

Drawing motivation from the above studies, the aim of the study is to investigate the simultaneous effects of the unsteady Couette flow past between two horizontal Riga plates with Hall and ion slip current with electromagnetic field. Explicit finite difference methodhas been used as a main tool to solve the problem. Also MATLAB R2015a has been used to calculate the results. The obtained results of different parameters have been shown graphically.

\section{Problem Formulation}

An incompressible laminar flow of viscous fluid between two horizontal parallel Riga plates has been considered, where one of which is moving and other is at rest. Let the lower plate be rest at $\tilde{y}=0$ and the upper plate is moving at a distance $\tilde{y}=d$ with a velocity $\frac{\pi v}{l}$. Let the direction of the flow be taken along the $\tilde{x}$-axis, $\tilde{y}$-axis is perpendicular to the flow and plates are parallel to the $\tilde{x} \tilde{z}$ - plane. As a generalized Couette flow, applied pressure gradient $\frac{\partial \tilde{p}}{\partial \tilde{x}}(=\mathrm{P})$ on the fluid is constant. Consider the plate is long enough in $x$-direction and the fluid motion is unsteady so that the fluid may be treated as two dimensional (i.e. $\left.\frac{\partial}{\partial \tilde{z}}=0\right)$. Therefore the continuity equation leads to $\tilde{u}=\tilde{u}(\tilde{y})$. Plates are fixed at two constant temperatures $T_{1}$ for the lower plate and $T_{2}$ for the upper plate, where $T_{2}>T_{1}$. The initial temperature of the fluid is assumed to be equal to the temperature of the lower plate $T_{1}$. Due to the effect of Hall and Ion-slip current, the generalized Ohm's law may state as follows:

$$
\mathbf{J}=\sigma(\mathbf{q} \wedge \mathbf{B})-\frac{\beta_{e}}{B_{0}}(\mathbf{J} \wedge \mathbf{B})+\frac{\omega_{e} \tau_{e} \beta_{i}}{B_{0}^{2}}(\mathbf{J} \wedge \mathbf{B}) \wedge \mathbf{B}
$$

Where, $\omega_{e} \tau_{e}=\beta_{e}$ may treat as Hall parameter. The physical model is shown in Fig.1.
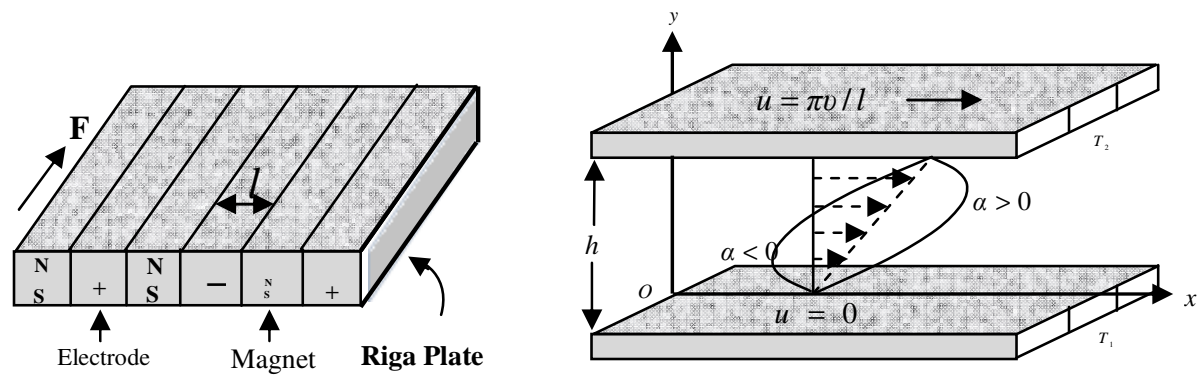

Fig.1: Physical model of the system 
A uniform magnetic force is generated by the Riga plate. The Lorentz force $\bar{f}=\mathbf{J} \wedge \mathbf{B} \approx \sigma(\mathbf{E} \wedge \mathbf{B})$ is defined as magnetic force. According to the Grinberg hypothesis this magnetic forces be defined as follows:

$$
\bar{f}=\mathbf{J} \wedge \mathbf{B}=\left(\frac{\pi}{8} J_{0} M_{0} e^{-\frac{\pi}{l} y}, 0, \frac{\pi}{8} J_{0} M_{0} e^{-\frac{\pi}{l} y}\right)
$$

Under the above assumptions of Couette flow and Bousniques approximations, it is found the dimensional forms of the momentum and energy equations are as follows:

$$
\begin{gathered}
\frac{\partial \tilde{u}}{\partial \tilde{t}}-v_{0} \frac{\partial \tilde{u}}{\partial \tilde{y}}=-\frac{\mathrm{P}}{\rho}+v \frac{\partial^{2} \tilde{u}}{\partial \tilde{y}^{2}}+\frac{\pi}{8 \rho}\left(1+\beta_{e}-\beta_{e} \beta_{\mathrm{i}}\right) J_{0} M_{0} e^{-\frac{\pi}{l} \tilde{y}} \\
\frac{\partial \tilde{w}}{\partial \tilde{t}}-v_{0} \frac{\partial \tilde{w}}{\partial \tilde{y}}=v \frac{\partial^{2} \tilde{w}}{\partial \tilde{y}^{2}}+\frac{\pi}{8 \rho}\left(1-\beta_{e}-\beta_{e} \beta_{\mathrm{i}}\right) J_{0} M_{0} e^{-\frac{\pi}{l} \tilde{y}} \\
\frac{\partial \tilde{T}}{\partial \tilde{t}}-v_{0} \frac{\partial \tilde{T}}{\partial \tilde{y}}=\frac{k}{\rho C_{p}} \frac{\partial^{2} \tilde{T}}{\partial \tilde{y}^{2}}+\frac{\mu}{\rho c_{p}}\left(\frac{\partial \tilde{w}}{\partial \tilde{y}}\right)^{2}
\end{gathered}
$$

The corresponding boundary conditions are

$$
\begin{gathered}
t \leq 0: \quad u=0, \quad w=, T=T_{1} \text { for all } y \geq 0 \\
t>0:\left\{\begin{array}{l}
u=0, \quad w=0, T=T_{1} \text { at } y=0 \\
u=\frac{\pi v}{l}, w=0, T=T_{2} \text { at } y=d
\end{array}\right.
\end{gathered}
$$

Similarity Analysis: To make the non-dimensional form of the equations (1)-(4), it is introduced the nondimensional variables are as follows:

$$
x=\frac{\pi}{l} \tilde{x}, \quad y=\frac{\pi}{l} \tilde{y}, \quad u=\frac{l}{\pi v} \tilde{u}, \quad w=\frac{l}{\pi v} \tilde{w}, t=\frac{\pi^{2} v}{l^{2}} \tilde{t}, \quad \theta=\frac{\tilde{T}-\tilde{T}_{1}}{\tilde{T}_{2}-\tilde{T}_{1}},
$$

Applying these into the equations (1)-(4), yields

$$
\begin{gathered}
\frac{\partial u}{\partial t}-V_{0} \frac{\partial u}{\partial y}=\alpha+\frac{\partial^{2} u}{\partial y^{2}}+H_{r}\left(1+\beta_{e}-\beta_{e} \beta_{\mathrm{i}}\right) e^{-y} \\
\frac{\partial w}{\partial t}-V_{0} \frac{\partial w}{\partial y}=\frac{\partial^{2} w}{\partial y^{2}}+H_{r}\left(1-\beta_{e}-\beta_{e} \beta_{\mathrm{i}}\right) e^{-y} \\
\frac{\partial \theta}{\partial t}-V_{0} \frac{\partial \theta}{\partial y}=\frac{1}{P_{r}} \frac{\partial^{2} \theta}{\partial y^{2}}-E_{c}\left(\frac{\partial w}{\partial y}\right)^{2}
\end{gathered}
$$

The corresponding boundary conditions are

$$
\begin{aligned}
& t \leq 0: \quad u=0, \quad \theta=0, \text { for all } y \geq 0 \\
& t>0: \begin{cases}u=0, & w=0, \quad \theta=0 \text { at } y=0 \\
u=1, & w=0, \theta \rightarrow 1, \text { at } y \rightarrow d\end{cases}
\end{aligned}
$$

where, $\alpha=-\frac{l^{3} \mathrm{P}}{\rho v^{2} \pi^{3}}$ is the dimensionless pressure gradient, $H_{r}=\frac{l^{3} J_{0} M_{0}}{8 \rho v^{2} \pi^{2}}$ is the modified Hartmann Number; $P_{r}=\frac{\rho c_{p} v}{k}$ is the Prandtl number ; $E_{c}=\frac{v^{2} \pi^{2}}{\left(\tilde{T}_{2}-\tilde{T}_{1}\right) c_{p} l^{2}}$ is the Eckert Number.

\section{Method of solution}

The system of non-dimensional coupled partial differential equations (5)-(7) have been solved by an explicit finite difference subject to the associated boundary conditions (8). It is considered maximum length of the plate is $x_{\max }(=10)$ and distance between the plates $d=2$ i.e. $y_{\max }=2$ as the lower plate is fixed at $y=0$. This

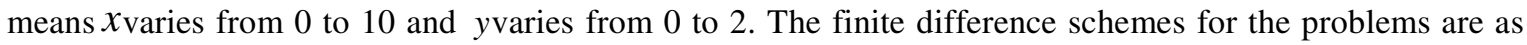
follows:

$$
\begin{aligned}
& \frac{u_{i, j}^{k+1}-u_{i, j}^{k}}{\Delta t}-V_{0} \frac{u_{i, j}^{k}-u_{i, j-1}^{k}}{\Delta y}=\alpha+\frac{u_{i, j+1}^{k}-2 u_{i, j}^{k}+u_{i, j-1}^{k}}{\Delta y^{2}}+H_{r}\left(1+\beta_{e}-\beta_{e} \beta_{\mathrm{i}}\right) e^{-y}{ }_{i} \\
& \frac{w_{i, j}^{k+1}-w_{i, j}^{k}}{\Delta t}-V_{0} \frac{w_{i, j}^{k}-w_{i, j-1}^{k}}{l}=\frac{w_{i, j+1}^{k}-2 w_{i, j}^{k}+w_{i, j-1}^{k}}{l^{2}}+H_{r}\left(1-\beta_{e}-\beta_{e} \beta_{\mathrm{i}}\right) e^{-y_{i}} \\
& \frac{\Theta_{i, j}^{k+1}-\Theta_{i, j}^{k}}{\Delta t}-V_{0} \frac{\Theta_{i, j}^{k}-\Theta_{i, j-1}^{k}}{\Delta y}=\frac{1}{P_{r}}\left(\frac{\Theta_{i, j+1}^{k}-2 \Theta_{i, j}^{k}+\Theta_{i, j-1}^{k}}{\Delta y^{2}}\right)-E_{c} \frac{w_{i, j}^{k}-w_{i, j-1}^{k}}{\Delta y}
\end{aligned}
$$


With the boundary conditions

$$
\begin{aligned}
& t \leq 0: \quad u=0, \theta=0, \text { forall } y \geq 0 \\
& t>0: \begin{cases}u=0, & w=0, \quad \theta=0 \text { at } y=0 \\
u=1, & w=0, \theta=1, \text { at } y=d\end{cases}
\end{aligned}
$$

Here, the subscripts $\mathrm{i}$ and $\mathrm{j}$ refer to $\mathrm{x}$ and $\mathrm{y}$ and the superscript $\mathrm{k}$ refers to time $\mathrm{t}$.

\subsection{Shear stresses and Nusselt number:}

The effects of pertinent parameters on the local and average shear stress from the velocity of the fluid have been investigated. The non-dimensional form of the local and average shear stress for the fluid is given by the relations $\tau_{L}=\left.\mu \frac{\partial u}{\partial y}\right|_{y=0}$ and $\tau_{A}=\left.\frac{1}{L} \int_{0}^{L} \mu \frac{\partial u}{\partial y}\right|_{y=0} d x$ and for the dust particle are given by $\tau_{p L}=\left.\mu \frac{\partial u_{p}}{\partial y}\right|_{y=0}$ and $\tau_{p A}=\left.\frac{1}{L} \int_{0}^{L} \mu \frac{\partial u_{p}}{\partial y}\right|_{y=0} d x$ respectively. The rate of heat transfer at the plate is defined as the Nusselt number. The local and average Nusselt number for the fluid are given by $N u_{L}=-\left.\mu \frac{\partial \theta}{\partial y}\right|_{y=0}$ and $N u_{A}=-\left.\frac{1}{L} \int_{0}^{L} \mu \frac{\partial \theta}{\partial y}\right|_{y=0} d x$.

\section{Results and Discussions}

Numerical results and the graphical presentations of the fluid velocity and temperature distributions have been explained for the influence of the relevant non-dimensional parameters namely pressure gradient parameter $(\alpha)$, modified Hartmann number $\left(H_{r}\right)$, Suction velocity parameter $\left(V_{0}\right)$, Hall parameter $\left(\beta_{e}\right)$, Ion-slip parameter $\left(\beta_{i}\right)$, Prandtl number $\left(P_{r}\right)$ and Eckert number $\left(E_{c}\right)$. The effects of those parameters on the necessary profiles are investigated with the fixed values of $\alpha=2.0, \beta_{e}=2.0, \beta_{i}=0.5, H_{r}=1.0, V_{0}=2.0, P_{r}=0.71$, and $E_{c}=0.5$.

\subsection{Steady-state solution:}

The behavior of the various entities on the velocity and temperature profiles has been elaborated graphically. Fig.2(a), Fig.2(b)andFig.2(c) illustrate the fluid velocities and temperature distributions $u, w$ and $\theta$ for different time $\tau$. The computations have been carried out for the different time such as $\tau=1,3,6$ and 8 with the time step size $\Delta t=0.0005$. It is observed from figures that there is no change after $\tau=8$ for $u, w$ and $\theta$. Fig.2(d)andFig.2(e) display the validity of the grid pairs on the velocity $u$ and temperature $\theta$. It has shown the velocity and temperature distributions for three grid pairs $(m, n)=(40,40),(m, n)=(45,45)$ and $(m, n)=(50,50)$ with time $\tau=8$ and time step $\Delta t=0.0005$. There is also negligible change among these grid pairs so that any one grid pair is acceptable to find the steady-state solution. It has seen that the same situation occur for the other distributions. The steady-state solution has performed for the values of $\tau>7$. In the present analysis, the following graphs have established for the choice of time $\tau=8$ with the grid pair $(m, n)=(50,50)$ and time step $\Delta t=0.0005$.

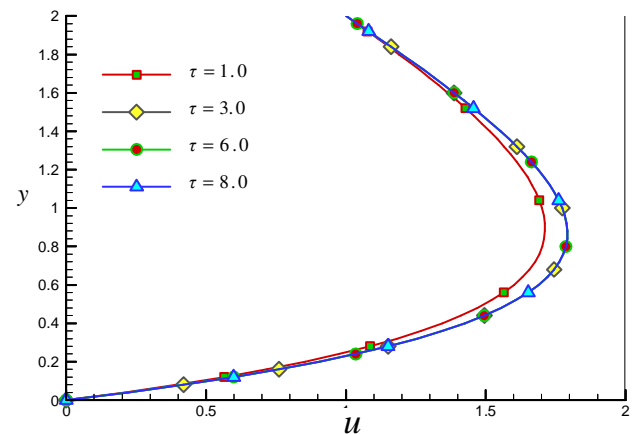

Fig.2(a): Time sensitivity on primary velocity $u$

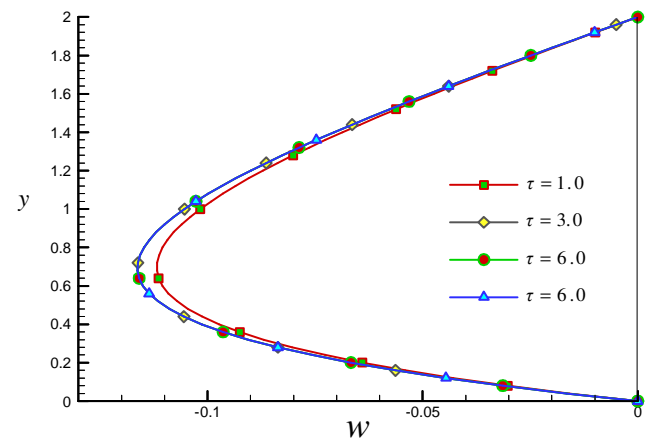

Fig.2(b): Time sensitivity on $w$ 


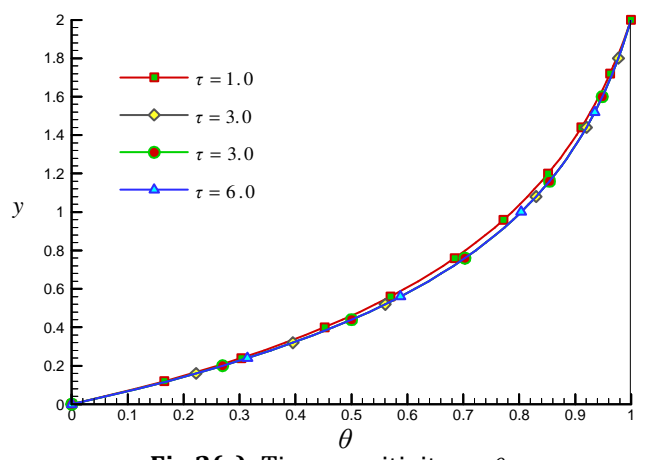

Fig.2(c): Time sensitivity on $\theta$

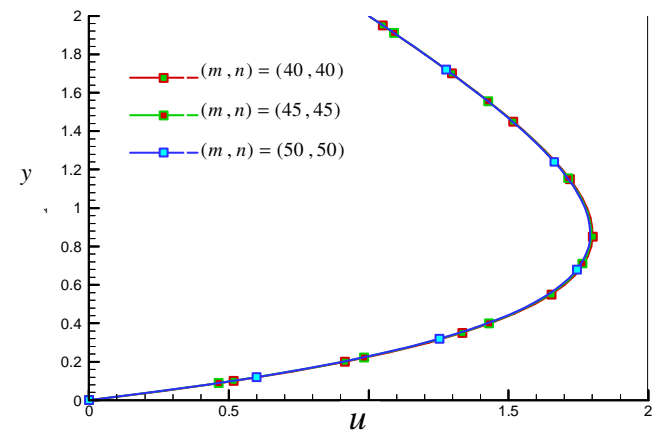

Fig.2(d): Mesh sensitivity on $u$

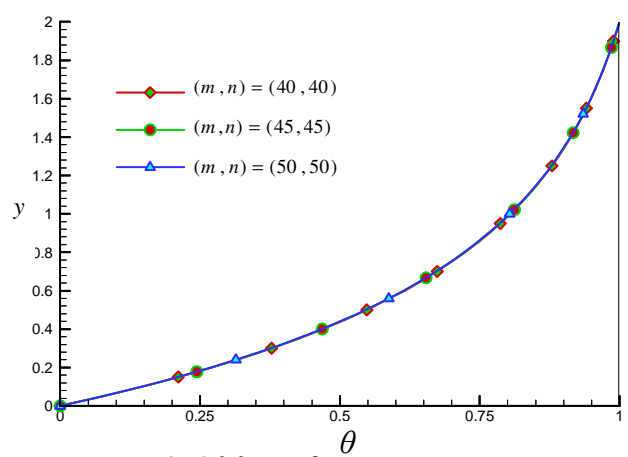

Fig.2(e): Mesh sensitivity on $\theta$

\subsection{Effects of various parameters}

To study the physical situation of the problem, it is mentioned that the figures(a), (b) and (c) of Fig.3to Fig.7 presented the distribution of velocity, local shear stress and average shear stress along the x-direction respectively.Figures (a), (b) and (c) ofFig.8 to Fig.11depicted the distribution of velocity, local shear stress and average shear stress along y-direction respectively. And figures (a), (b) and (c) of Fig.12to Fig.17 represented the distribution of temperature, local Nusselt number and average Nusselt number respectively.

The effects of dimensionless pressure gradient $\alpha$ on the velocity is shown in Figs.3(a)-(c).Since $\alpha=-l^{3} \mathrm{P} /\left(\rho v^{2} \pi^{3}\right)$, it means that $\alpha>0$ whenever constant pressure gradient $\mathrm{P}$ decreasing in the direction of motion; in that case, the velocity $u$ has increasing effect with the increase of $\alpha$ over the entire width between the plates. But $\alpha<0$ influence that $\mathrm{P}$ increasing in the direction of motion and the velocity $u$ has occurred back-flow, which have shown in Fig.3(a). The same situation occurred for the local and average shear stress on the fluid which have shown in Figs.3(b)and(c) respectively.

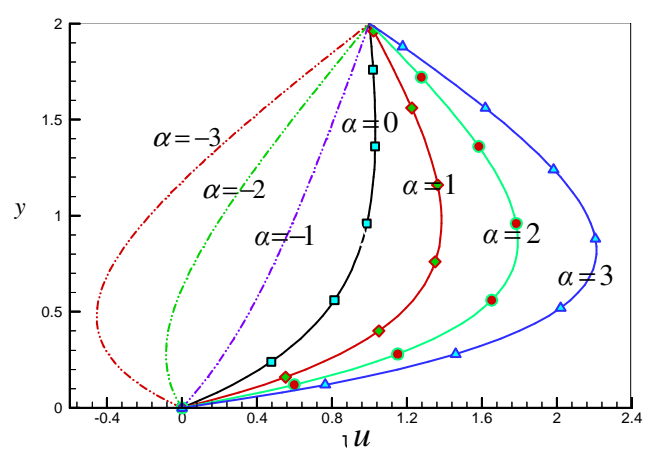

Fig.3(a): Effect of $\alpha$ on $u$

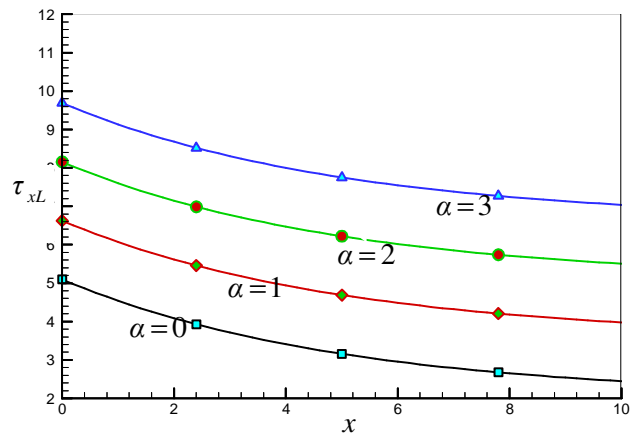

Fig.3(b): Effect of $\alpha$ on $\tau_{x L}$ 


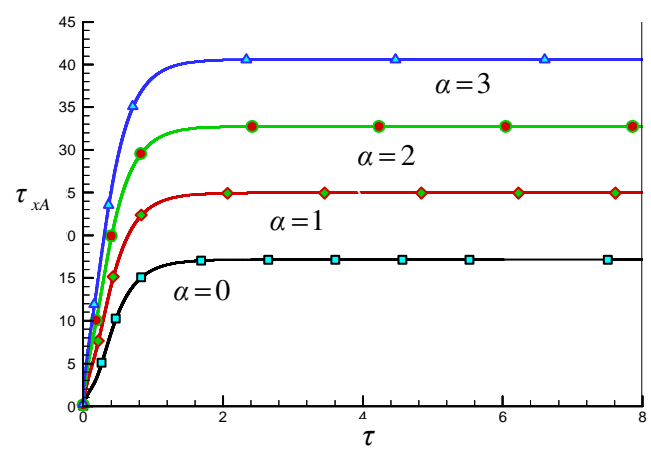

Fig.3(c): Effect of $\alpha$ on $\tau_{x A}$

In the figures, Figs.4(a)-(c) represented the performance of Hall parameter $\beta_{e}$ on the primary velocity $u$, local shear stress $\tau_{x L}$ and on the average shear stress $\tau_{x A}$ respectively. It is showed that the primary velocity $u$, local and average shear stress $\tau_{x L}$ and $\tau_{x A}$ are all increasing with the increase of $\beta_{e}$.

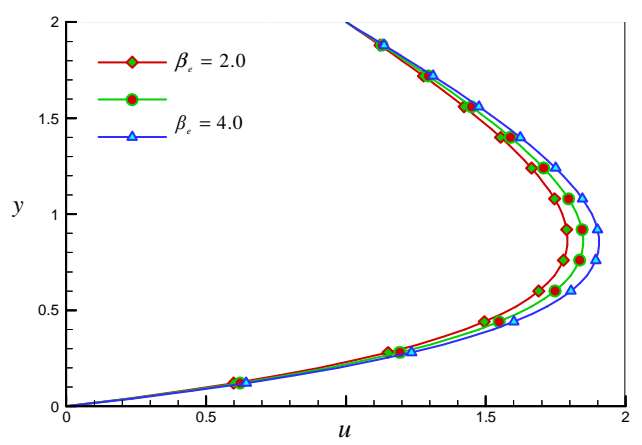

Fig.4(a): Effect of $\beta_{e}$ on $u$

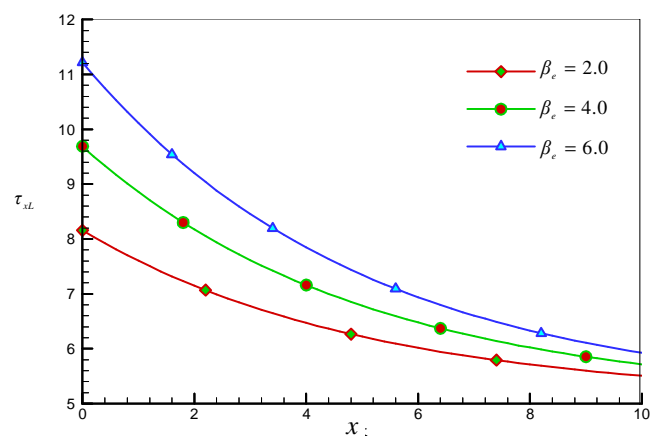

Fig.4(b): Effect of $\beta_{e}$ on $\tau_{x L}$

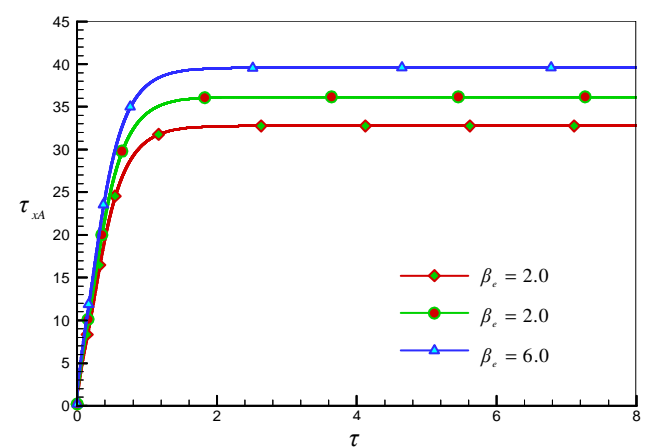

Fig.4(c): Effect of $\beta_{e}$ on $\tau_{x A}$

The impact of Ion-slip parameter $\beta_{i}$ on the velocity $u$, local shear stress $\tau_{x L}$ and the average shear $\tau_{x A}$ stress are displayed respectively in Figs.5(a)-(c). It is observed that the primary velocity $u$, local and average shear stress $\tau_{x L}$ and $\tau_{x A}$ are all decreased with the increase of $\beta_{i}$. 


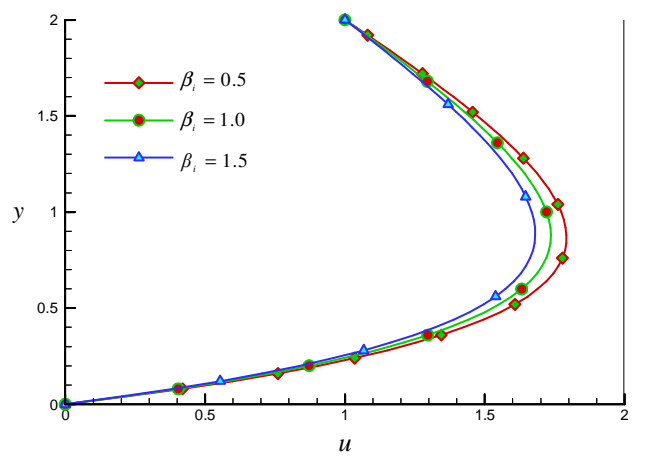

Fig.5(a): Effect of $\beta_{i}$ on $u$

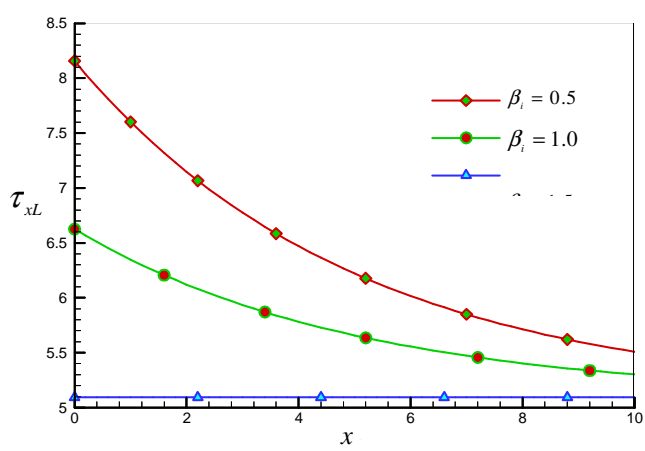

Fig.5(b): Effect of $\beta_{i}$ on $\tau_{x L}$

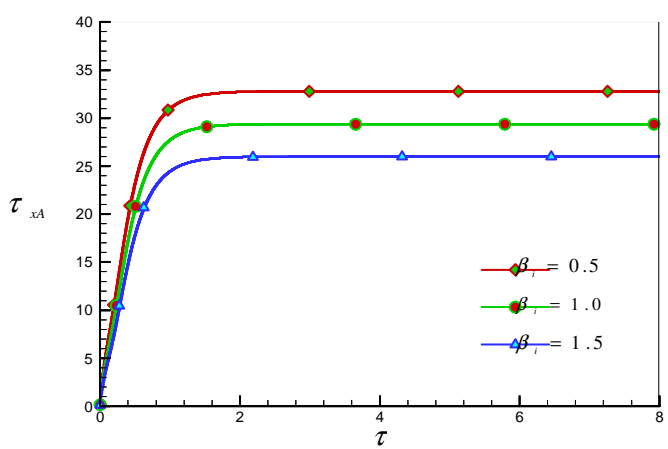

Fig.5(c): Effect of $\beta_{i}$ on $\tau_{x A}$

Figs.6(a)-(c) depict the effect of modified Hartmann number $H_{r}$ on the velocity $u$, local shear stress $\tau_{L}$ and on the average shear stress $\tau_{A}$ respectively. It is evident that $u, \tau_{x L}$ and $\tau_{x A}$ are all rises with the influence of $H_{r}$.

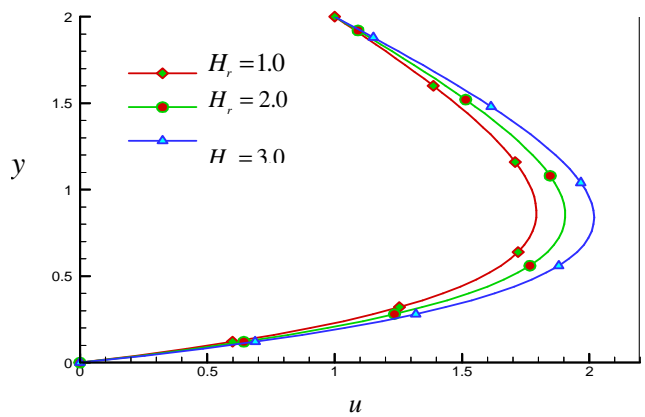

Fig.6(a): Effect of $H_{r}$ on $u$

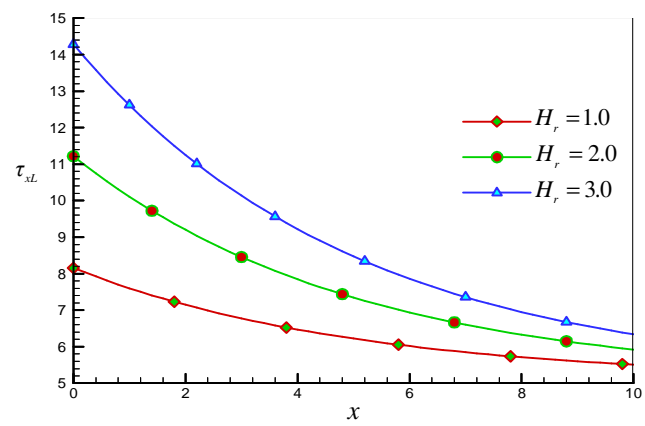

Fig.6(b): Effect of $H_{r}$ on $\tau_{x L}$

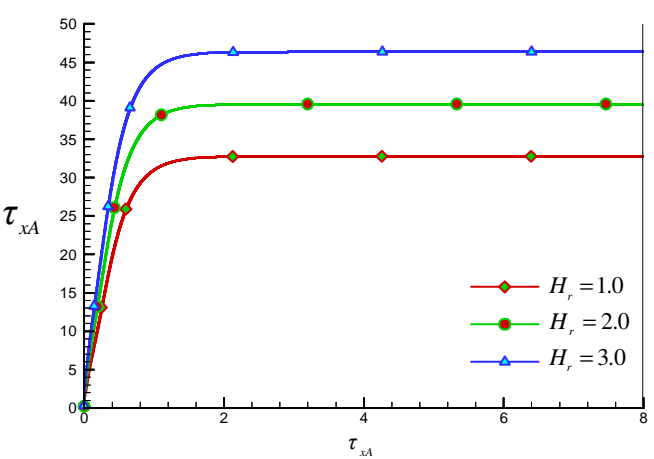

Fig6(c): Effect of $H_{r}$ on $\tau_{x A}$ 
Characteristics of the Suction velocity $v_{0}$ on the velocity $u$, local shear stress $\tau_{x L}$ and on the average shear stress $\tau_{x A}$ are presented in Figs.7(a)-(c) respectively. In Fig.7(a), it is observed that the velocity $u$ has a cross flow where the velocity has increasing effect within the interval $0<y<1$ (approx.) thereafter it has decreasing effect, whereas $\tau_{x L}$ and $\tau_{x A}$ are increased with the increasing effect of $V_{0}$.

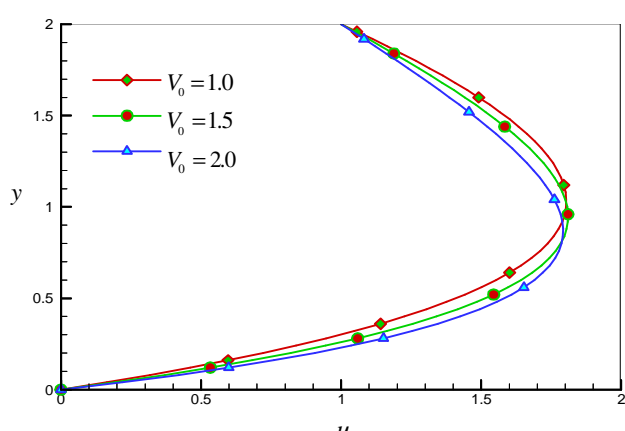

Fig.7(a): Effect of $V_{0}$ on $u$

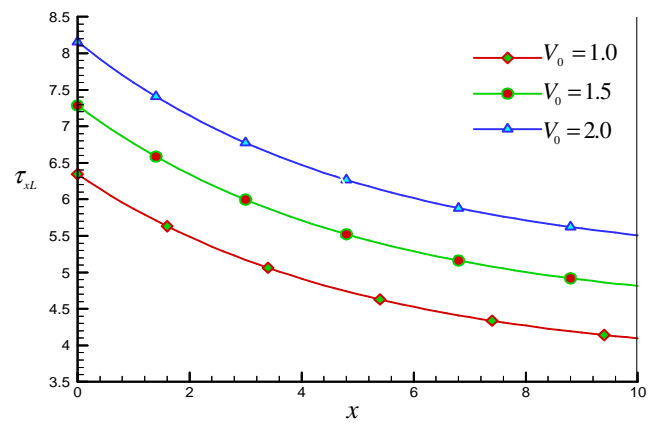

Fig.7(b): Effect of $V_{0}$ on $\tau_{x L}$

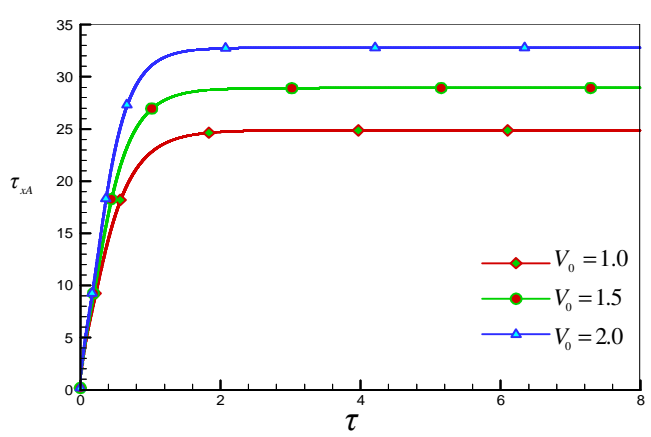

Fig.7(c): Effect of $V_{0}$ on $\tau_{x A}$

Figs.8(a)-(c) explain the influence of Hall current parameter $\beta_{e}$ on the secondary velocity $w$, local shear stress $\tau_{z L}$ and on the average shear stress $\tau_{z A}$ respectively. It has shown that increasing values of $\beta_{e}$ the secondary velocity $w$, local shear stress $\tau_{z L}$ and average shear stress $\tau_{z \mathrm{~A}}$ become decreases.

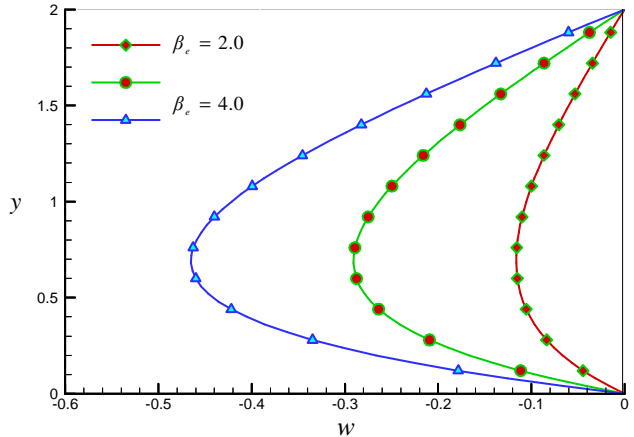

Fig.8(a): Effect of $\beta_{e}$ on $w$

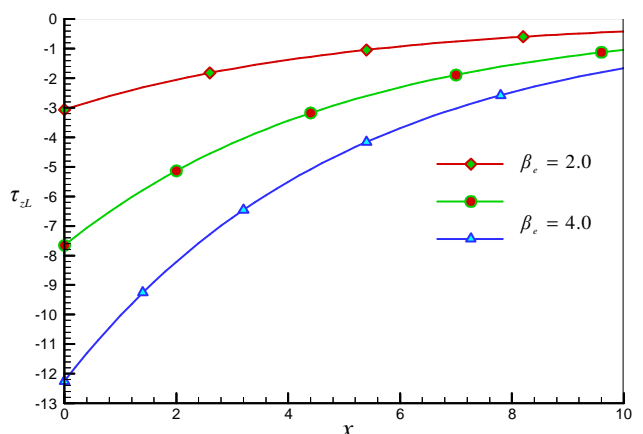

Fig.8(b): Effect of $\beta_{e}$ on $\tau_{z L}$ 


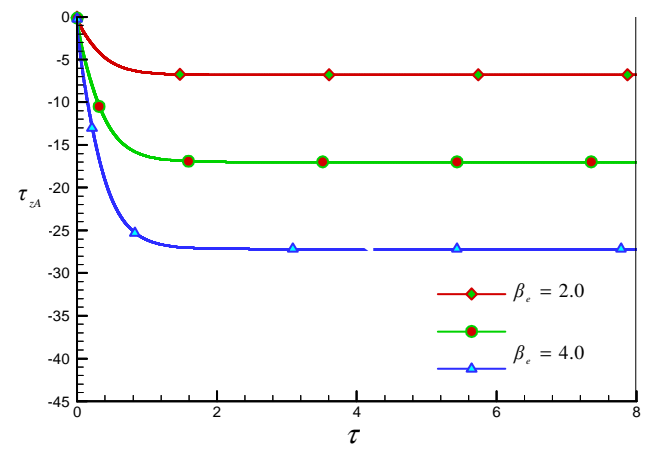

Fig.8(c): Effect of $\beta$ on $\tau_{z A}$

The effects of Ion-slip parameter $\beta_{i}$ on the secondary velocity profile $w$, local shear stress $\tau_{z L}$ and on the average shear stress $\tau_{z A}$ are exposed inFigs.9(a)-(c) respectively. It has shown for the increasing values of $\beta_{i}$, the secondary velocity profile $w$, local and average shear stress $\tau_{z L}$ and $\tau_{z A}$ are decreased.

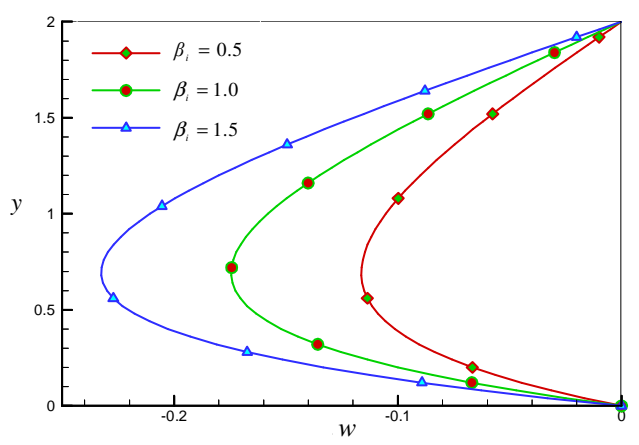

Fig.9(a): Effect of $\beta_{i}$ on $w$

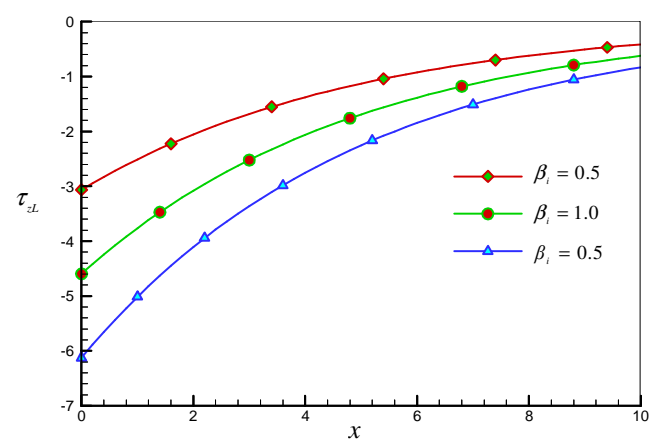

Fig.9(b): Effect of $\beta_{i}$ on $\tau_{z I}$

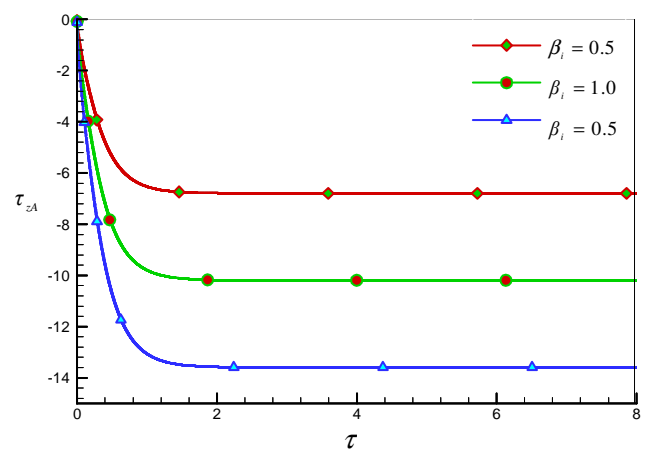

Fig.9(c): Effect of $\beta_{i}$ on $\tau_{7 A}$

The variations of modified Hartman number $H_{r}$ on the secondary velocity $w$, local shear stress $\tau_{z L}$ and on the average shear stress $\tau_{z A}$ are depicted in Figs.10(a)-(c). With the increasing effect of $H_{r}$ it has found that $w, \tau_{z L}$ and $\tau_{z A}$ are decreased. 


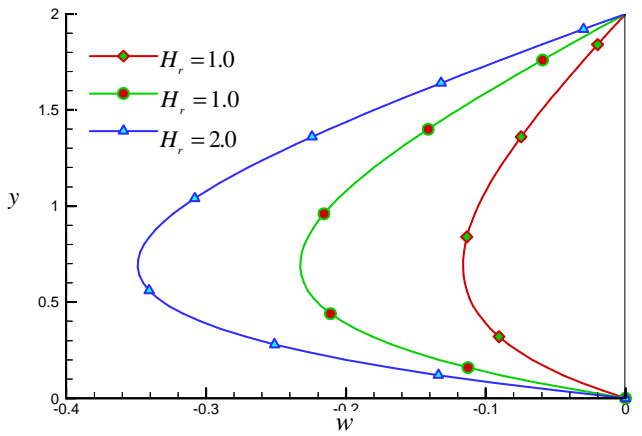

Fig.10(a): Effect of $H_{r}$ on $w$

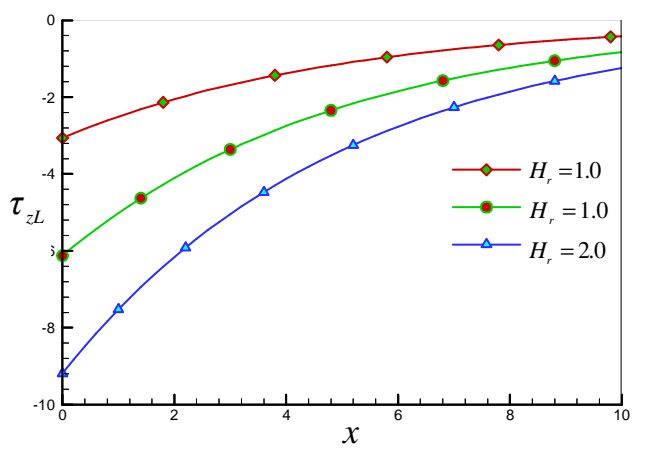

Fig.10(b): Effect of $H_{r}$ on $\tau_{z L}$

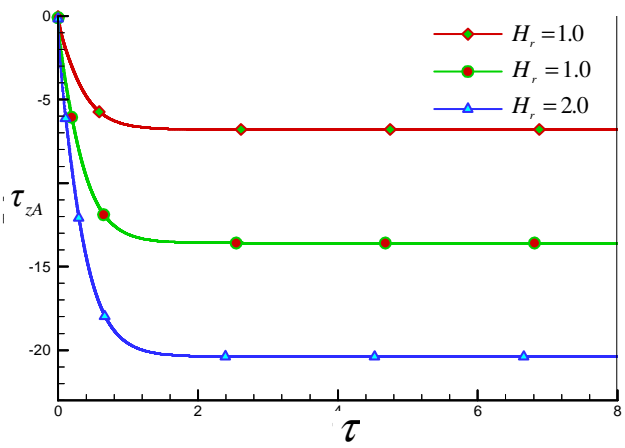

Fig.10(c): Effect of $H_{r}$ on $\tau_{z A}$

Figs.11(a)-(c) are plotted to explain the behavior of suction velocity $V_{0}$ on the velocity $w$, local shear stress $\tau_{z L}$ and the average shear stress $\tau_{z A}$ respectively. It has shown that the back flow of $w$ with the increasing values of suction velocity $V_{0}$, whereas $\tau_{z L}$ and $\tau_{z A}$ are displayed decreasing effect.

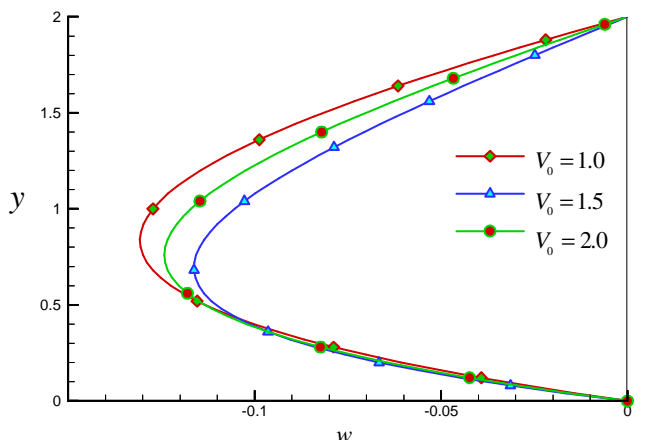

Fig.11(a): Effect of $V_{0}$ on $w$

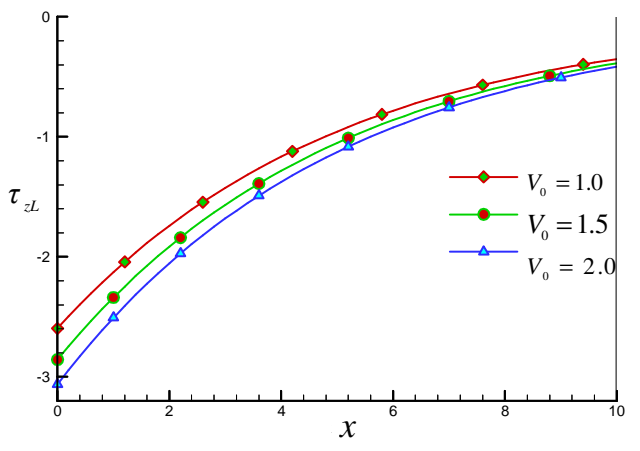

Fig.11(b): Effect of $V_{n}$ on $\tau_{z L}$

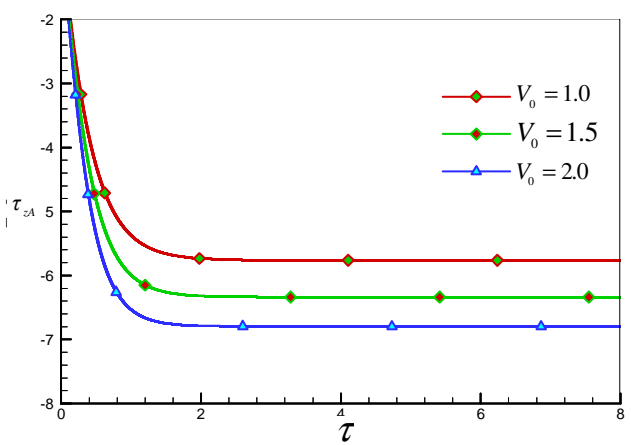

Fig.11(c): Effect of $V_{0}$ on $\tau_{z A}$ 
To find the variations of Hall current parameter $\beta_{e}$ on the temperature $\theta$, local Nusselt number $N u_{L}$ and on the average Nusselt number $N u_{A}$ have been demonstrated in Figs.12(a)-(c) respectively. It is found from the figures that for the increasing effect of $\beta_{e}$, temperature $\theta$ is increased whereas $N u_{L}$ and $N u_{A}$ showed the inverse effects.

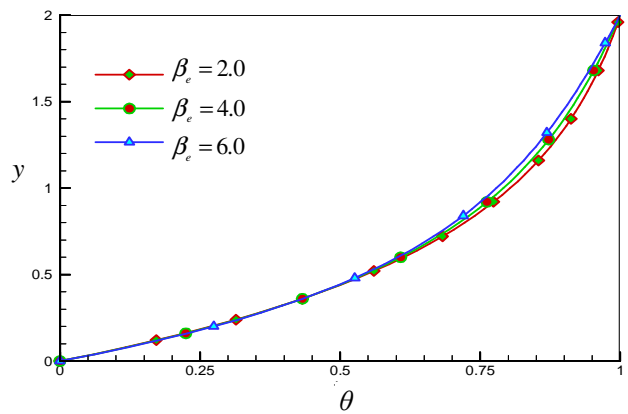

Fig.12(a): Effect of $\beta_{e}$ on $\theta$

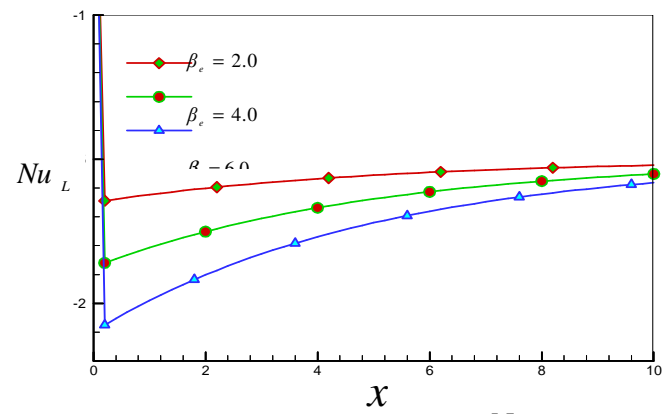

Fig.12(b): Effect of $\beta_{e}$ on $N u_{L}$

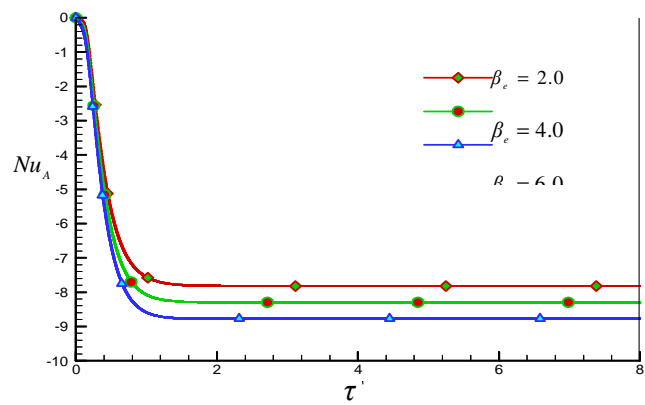

Fig.12(c) :Effect of $\beta_{e}$ on $N u_{A}$

The effects of Ion-slip current parameter $\beta_{i}$ on the temperature $\theta$, local Nusselt number $N u_{L}$ and on the average Nusselt number $N u_{A}$ are plotted in Figs.13(a)-(c) respectively. It has shown that for the increasing effect of $\beta_{i}$, temperature $\theta$ is increased whereas $N u_{L}$ and $N u_{A}$ showed the decreasing effects.

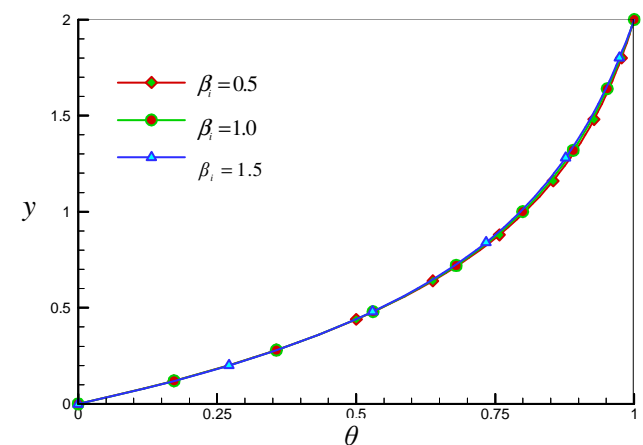

Fig.13(a):Effect of $\beta_{i}$ on $\theta$

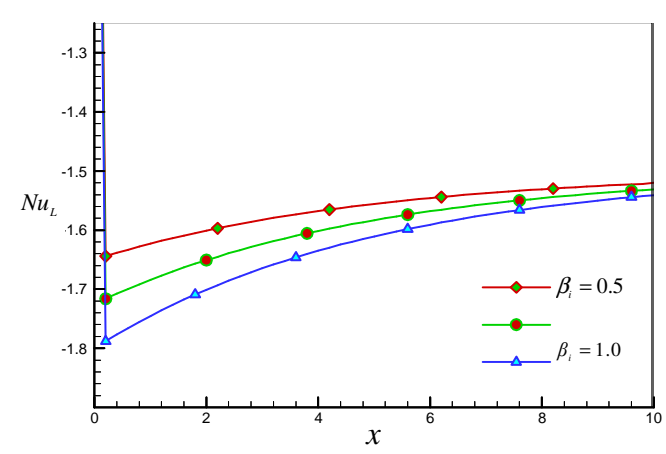

Fig.13(b): Effect of $\beta_{i}$ on $N u_{L}$

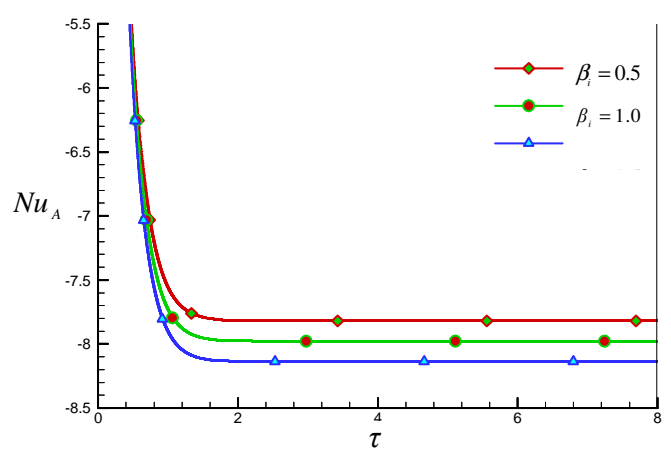

Fig.13(c): Effect of $\beta_{i}$ on $N u_{A}$ 
It is visualized from the figures Figs.14(a)-(c) that for increasing values of modified Hartman number $H_{r}$ on the temperature $\theta$ depicted rising effect and the Nusselt number $N u_{L}$ and the average Nusselt number $N u_{L}$ are demonstrated decreasing effects.

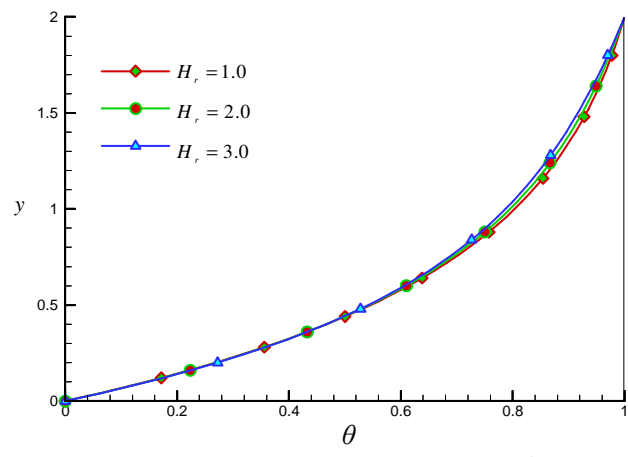

Fig.14(a): Effect of $H_{r}$ on $\theta$

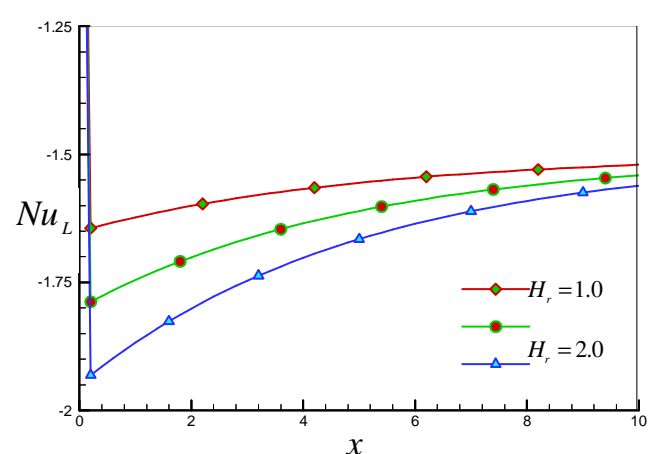

Fig.14(b): Effect of $H_{r}$ on $N u_{L}$

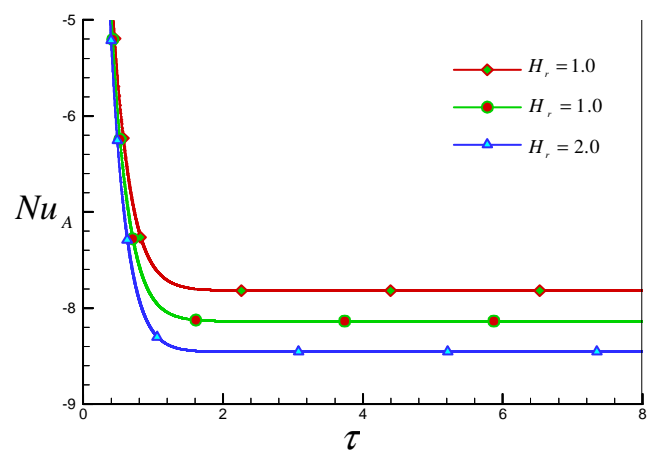

Fig.14(c): Effect of $H_{r}$ on $N u_{A}$

Temperature profile $\theta$ is increased due to the increasing effect of Prandtl number $P_{r}$ that is seen in Figs.15(a) whereas Figs.15 (b) and Figs.15 (b) exhibit the reverse effect of $\theta$.

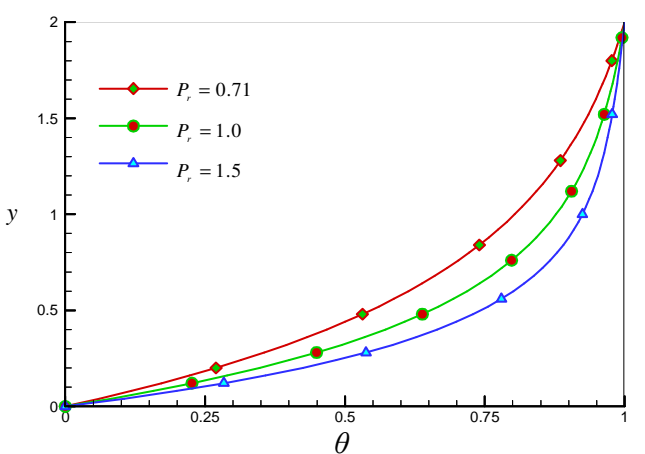

Fig.15(a): Effect of $P_{r}$ on $\theta$

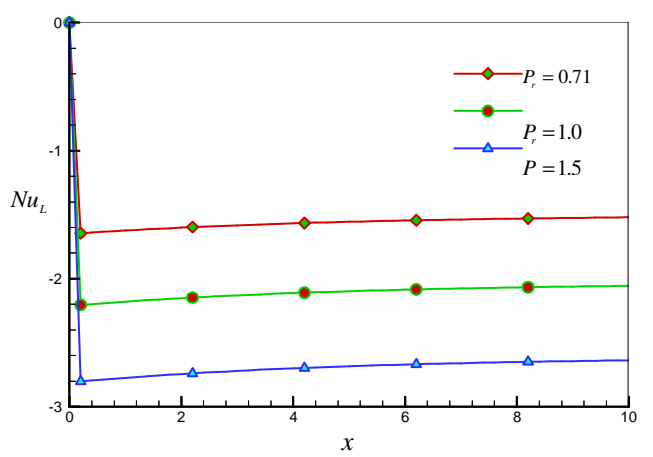

Fig.15(b): Effect of $P_{r}$ on $N u_{L}$ 


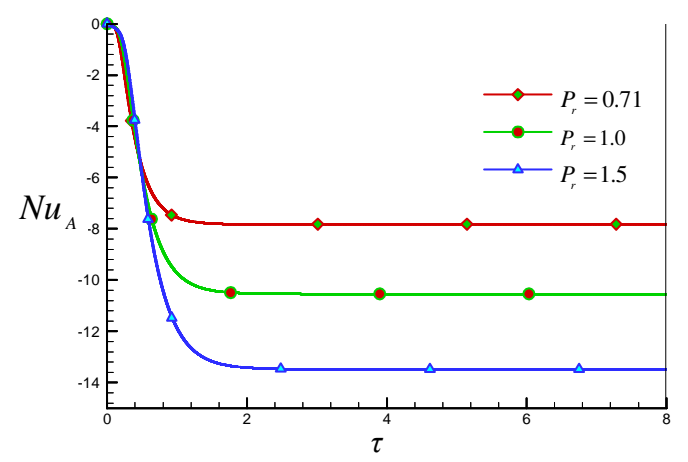

Fig.15(c): Effect of $P_{r}$ on $N u_{A}$

It is observed from Figs.16(a)-(c) the effects of Eckert number $E_{c}$ on the temperature $\theta$, local Nusselt number $N u_{L}$ and on the average Nusselt number $N u_{L}$ respectively. It has shown that $\theta$ depicts the increasing effect but $N u_{L}$ and $N u_{L}$ show the decreasing effect with the effects of Eckert number.

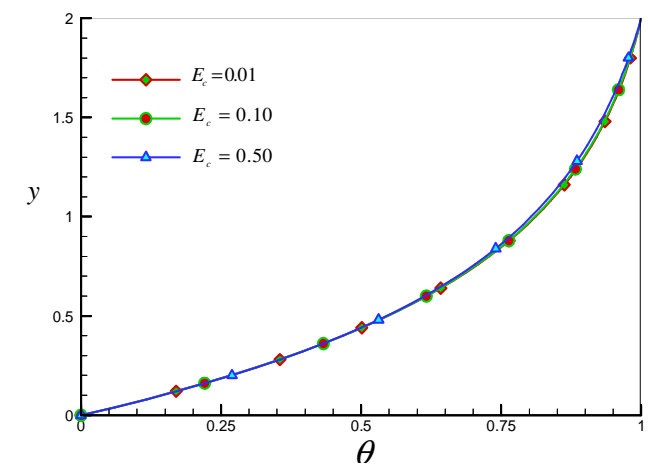

Fig.16(a): Effect of $E_{c}$ on $\theta$

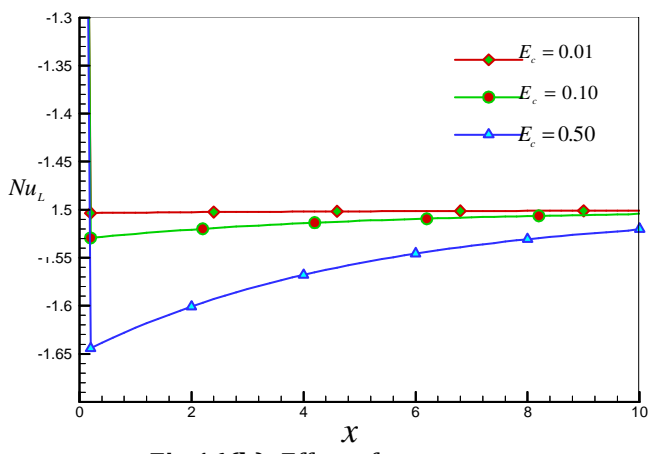

Fig.16(b): Effect of $E_{c}$ on $N u_{L}$

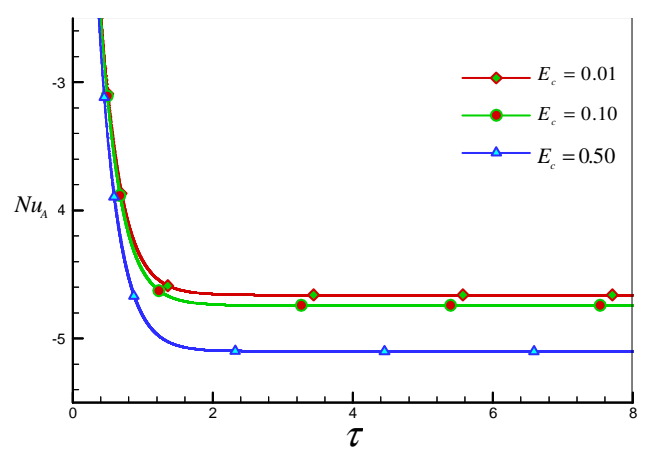

Fig.16(c):Effect of $E_{c}$ on $N u_{A}$

The effects of the suction velocity $V_{0}$ on the temperature $\theta$, local Nusselt number $N u_{L}$ and on the average Nusselt number $N u_{L}$ are plotted in Figs.17(a)-(c) respectively. It has shown that with the increasing effect of $V_{0}$, the temperature $\theta$, local and average $N u_{L}$ and $N u_{L}$ showed decreasing effects. 


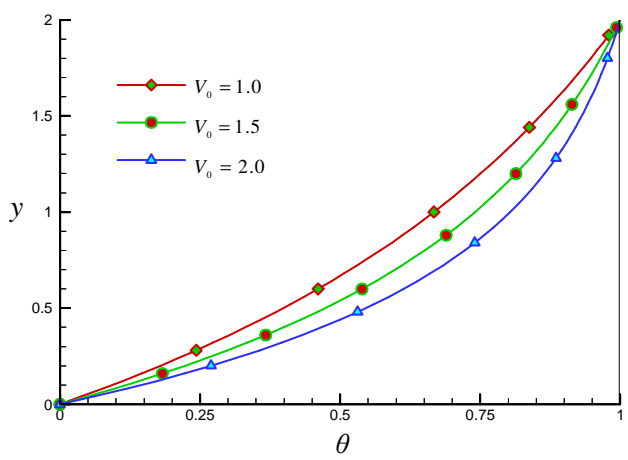

Fig.17(a): Effect of $V_{0}$ on $\theta$

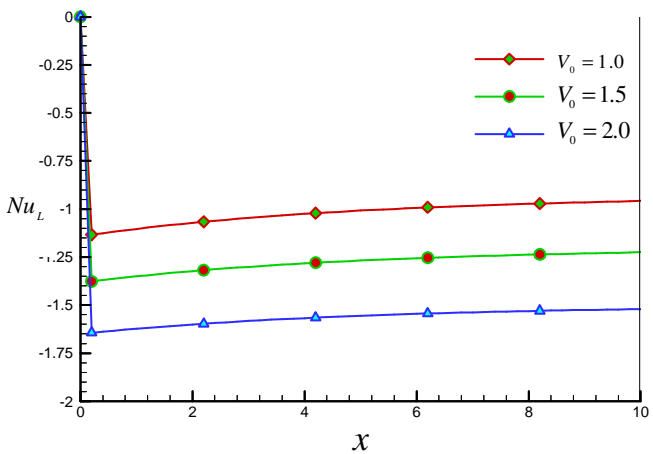

Fig.17(b): Effect of $V_{0}$ on $N u_{L}$

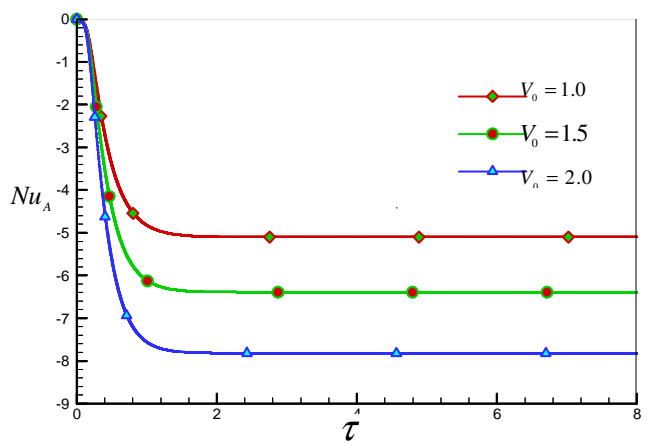

Fig.17(c):Effect of $V_{0}$ on $N u_{A}$

\section{Conclusions}

The characteristics of two horizontal parallel Riga plates in the presence of Hall and ion slip currents effects have been investigated numerically. The main findings of the study are as follows:

i. Primary velocity profile $u$ increases for increasing values of the $\alpha, \beta_{e}$ and $H_{r}$, while it has decreasing effects with the increase of $\beta_{i}$. The suction velocity $V_{0}$ has a cross effect where the skin friction is increased as the suction velocity $V_{V_{0}}$ is increased.

ii. Secondary velocity profile $w$ decreases for increasing values of the $\beta_{e}, \beta_{i}$ and $H_{r}$. The suction velocity $V_{0}$ shows a cross effect where the skin friction coefficient is increased as the suction velocity $V_{0}$ is decreased.

iii. The temperature profile increases with the rising values of the $\beta_{e}, \beta_{i}$ and $H_{r}$ where the local and average Nusselt numbers shows the reverse effects.

\section{Nomenclature}

$\tilde{u}$ : Velocity components along $\tilde{x}$-axis $\tilde{v}$ : Velocity components along $\tilde{y}$-axis

$\tilde{w}$ : Velocity components along $\tilde{z}$ - axis

$\tilde{t}$ : Dimensional time

$\beta_{e}:$ Hall parameter

$\beta_{i}$ : Ion-slip parameter

$\alpha$ : Dimensionless pressure gradient $\mathrm{k} \quad$ : Thermal conductivity of the fluid

$c_{p}$ : Specific heat capacity at constant pressure

$J_{0}:$ Current density

$\tilde{T}$ : Temperature of the fluid

$\tilde{T}_{w}$ : Constant temperature near the plate

$\tilde{T}_{\infty}$ : Temperature outside of the boundary layer

$\Delta t:$ Time increment

$t$ : Dimensionless time 


\author{
$\rho$ : Density of the fluid \\ $\sigma$ : Conductivity of the fluid \\ $\tau$ : Maximum time \\ $E_{c}:$ Eckert number \\ $v$ : Kinematic viscosity of the fluid \\ $\mu$ : Coefficient of viscosity of the fluid \\ $u$ : Dimensionless velocity component in $\mathrm{x}$-axis \\ $v$ : Dimensionless velocity component in y-axis
}

\author{
$\theta$ : Dimensionless temperature \\ $H_{r}$ : Hartmann number \\ $P_{r}:$ Prandtl number \\ $\tau_{L}$ : Local shear stress \\ $N u_{\text {: }}$ Local Nusselt number \\ $\tau_{A}:$ Average shear stress \\ $N u_{A}$ : Average Nusselt number
}

\section{References}

[1] A. Gailitis, and O. Leilausis, On a possibility to reduce the hydro dynamical resistance of a plate in an electrode. Appl. Magnetohydrodyn, Vol.12, pp. 143-146, 1961.

[2] Timothy W. Berger, John Kim, Changhoon Lee and Junwoo Lim, Turbulent boundary layer control utilizing the Lorentz force, Physics of Fluids 12, 631 ,2000; https://doi.org/10.1063/1.870270.

[3] A. Pantokratoras, E. Magyari, MHD free-convection boundary layer flow from a riga plate. J.Eng. Maths, Vol.64(3), pp. 303-315, 2009.

[4] L.Wahidunnisa, K.Subbarayudu, S.Suneetha, Effect of viscous dissipation over a Riga plate in a nano fluid with heat source/sink. IJTIMES, Vol.4, Issue.6, 2016.

[5] Z. Iqbal, EhtshamAzhar, ZaffarMehmood and E.N. Maraj, Melting heat transport of nanofluidic problem over a Riga plate with erratic thickness: Use of Keller Box scheme, Results in Physics, Vol.7, pp.36483658, 2017.

[6] Ayub M, Abbas T, Bhatti MM., Inspiration of slip effects on electromagneto hydrodynamics (EMHD) nano fluid flow through a horizontal Riga plate. EurPhys J Plus 16193, 2016.

[7] Adeel Ahmed, SaleemAsghar and SumairaAfzal, Flow of nanofluid past a Riga plate. Journal of Magnetisnand Magnetic Materials.Vol.420, pp. 44-48, 2016.

[8] Emad M. Aboeldahab and Elsayed M. E. Elbarbary, Hall current effect on magneto-hydrodynamic free convection flow past a semi-infinite vertical plate with mass transfer. Int. J. Engg. Sci.,Vol 39, pp.16411652, 2001.

[9] V. Javeri, Combined influence of Hall effect, ion slip, viscous dissipation and Joule heating on MHD heat transfer in a channel.Vol.8, pp.193-202,1975.

[10] A.H. Eraslan, Temperature distributions in MHD channels with Hall Effect. AIAAJ, Vol.7, pp.186 $188,1969$.

[11] Mohamed A. Seddeek and Emad M. Aboeldahab, Radiative effects on unsteady MHD free convection with Hall current near an infinite vertical porous plate.IJMMS, Vol.26, pp.249-255, 2001.

[12] P.V. SatyaNarayana, G. Ramireddy and S. Venkataramana, Hall Current Effects on Free Convection MHD Flow Past a Porous Plate. IJAME,Vol. 3, pp. 350-363, ISSN: 2180-1606, 2011.

[13] LokenathDebnath, Sukomal Chandra Ray and Anddi Kumar Chatterjee, Effects of Hall Current on Unsteady Hydromagnetic Flow past a Porous Plate in a Rotating Fluid System.ZAMM,Vol.59, pp.469-471, 1979.

[14] M. A. Al-Nimr and S. Masoud, Unsteady free convection flow over a vertical flat plateimmersed in a porous medium, Fluid Dynamics Research, Vol.23, pp.153-160, 1998.

[15] M. VeeraKrishna , N. AmeerAhamad, Ali J. Chamkha ,Hall and ion slip effects on unsteady MHD free convective rotating flow through a saturated porous medium over an exponential accelerated plate.Alexandria Engineering Journal , Vol.59, pp.565-577, 2020.

[16] Bhpendra Kumar Sharma, Abhay Kumar Jha, R.C. Chaudhary, Hall effect on MHD mixed convective flow of a viscous incompressible fluid past a vertical porous plate immersed in porous medium with heat source /sink. Romanian Journal of Physics, Vol.52, pp.5-7, 2007.

[17] D. Angirasa and G.P. Peterson, Natural Convection Heat Transform from an Isothermal Vertical Surface to a Fluid Saturated Thermally Stratified Porous Medium. Int. J. Heat Mass Transfer, Vol. 14(8), pp. 4329-4335,1997.

[18] B.V.R.Kumar, P. Singh, Effect of Thermal Stratification on Free Convection in a Fluid Saturated Porous Enclosure. Numer. Heat Transfer, Vol. 34, pp. 343-356, 1999. 
[19] NirmalGhara, SovanLalMaji, Sanatan Das, Rabindranath Jana, Swapan Kumar Ghosh, Effects of Hall Current and Ion-Slip on Unsteady MHD Couette Flow. Open Journal of Fluid Dynamics, Vol.2, pp.1-13, 2012.

[20] Hazem Ali Attia, Unsteady Couette Flow with Heat Transfer in a Viscoelastic Fluid Considering the Ion Slip. Journal of the Korean Physical Society,Vol. 47, No. 5, pp. 809-817, 2005.

[21] Hazem A. Attia , Unsteady MHD Couette Flow with Heat Transferin the Presence of Uniform Suction and Injection. Mechanics and Mechanical Engineering, Vol. 12, No. 2, pp. 165-176, 2008.

[22] V.Ravi Kumar, M.C.Raju ,G.S.S.Raju,MHD Three Dimensional Couette Flow past aPorous Plate with Heat Transfer. IOSR Journal of Mathematics (IOSRJM), ISS

N: 2278-5728 Vol. 1, Issue 3, pp. 03-09, 2012.

[23] IsahBalaYabo, Basant Kumar Jha, Jeng-EngLin,On a Couette Flow of Conducting Fluid. International Journal of Theoretical and Applied Mathematics, Vol. 4(1), pp.8-21, 2018.

[24] S HarisinghNaik, M V Ramana Murthy, K. Rama Rao ,The effect of Hall current on an unsteady MHD free convectiveCouette flow between two permeable plates in the presence of thermal radiation. IJCER, ISSN 2250 - 3005, Vol. 04, Issue. 7, 2014.

[25]Victor M. Job and SreedharaRaoGunakala, Unsteady MHD Free Convection Couette FlowThrough a Vertical Channel in the Presence of Thermal Radiation With Viscous and Joule Dissipation Effects: Using Galerkin's FiniteElement Method. IJAIEM, Vol.2, Issue 9, ISSN 2319 -4847, 2013. 
Figures
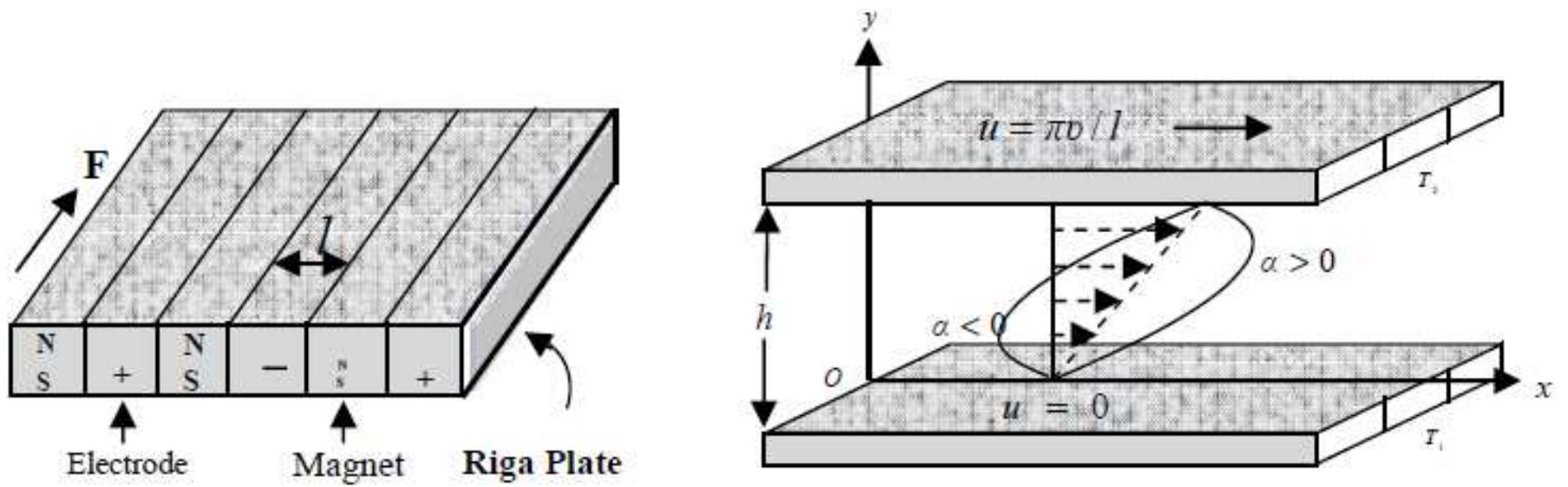

Figure 1

See the Manuscript Files section for the complete figure caption. 


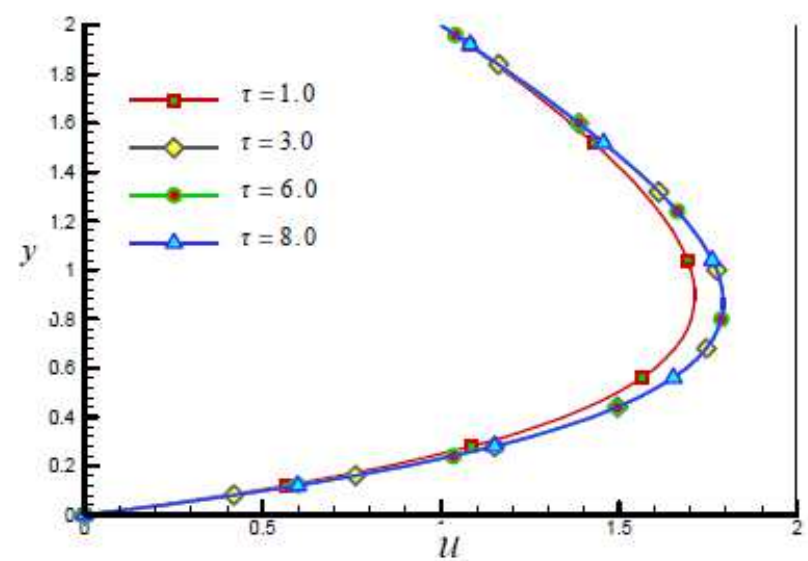

(a): Time sensitivity on primary velocity $u$

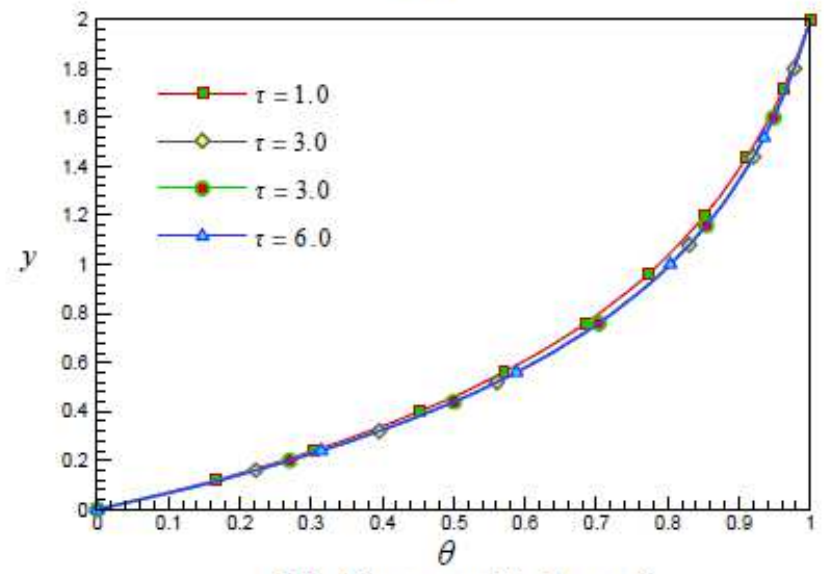

(c): Time sensitivity on $\theta$

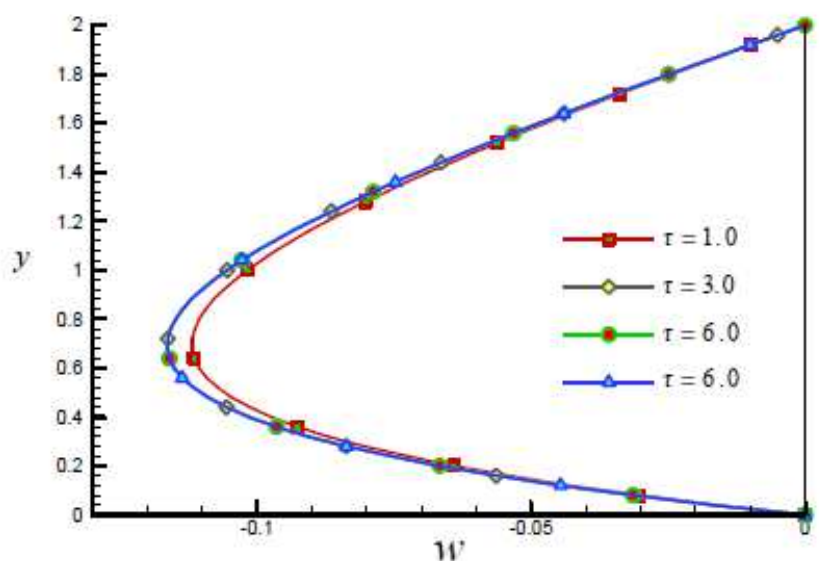

(b): Time sensitivity on $w$

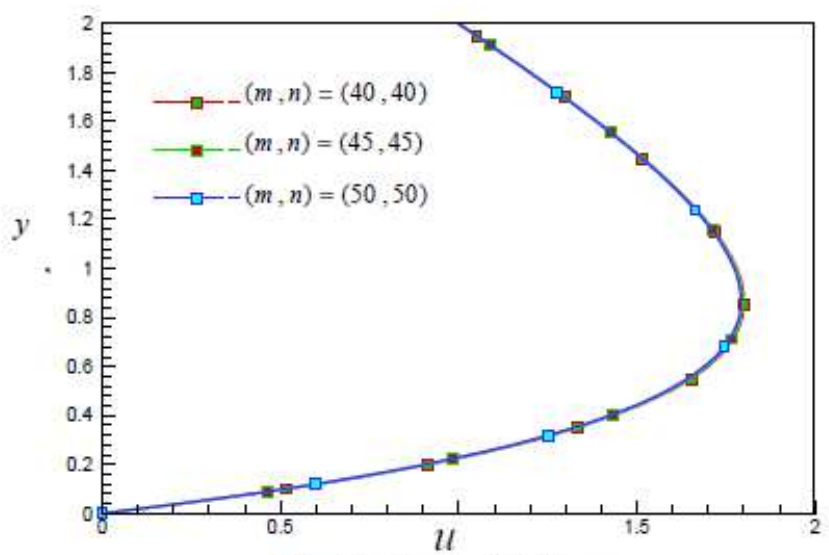

(d): Mesh sensitivity on $u$

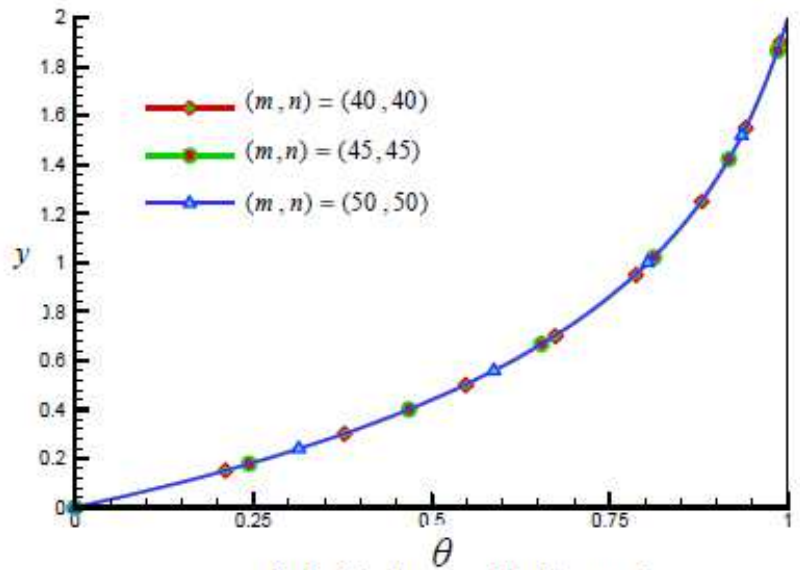

(e): Mesh sensitivity on $\theta$

\section{Figure 2}

See the Manuscript Files section for the complete figure caption. 


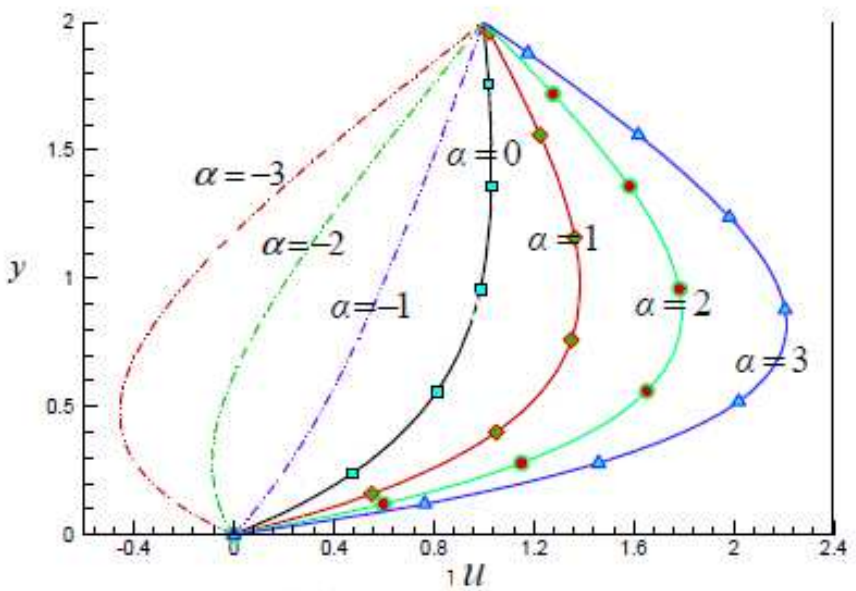

(a): Effect of $\alpha$ on $u$

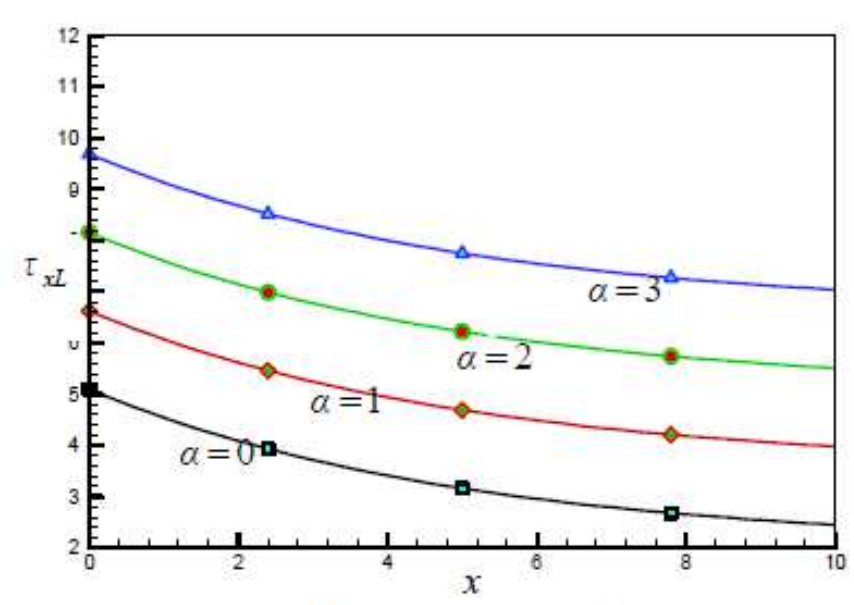

(b): Effect of $\alpha$ on $\tau_{x L}$

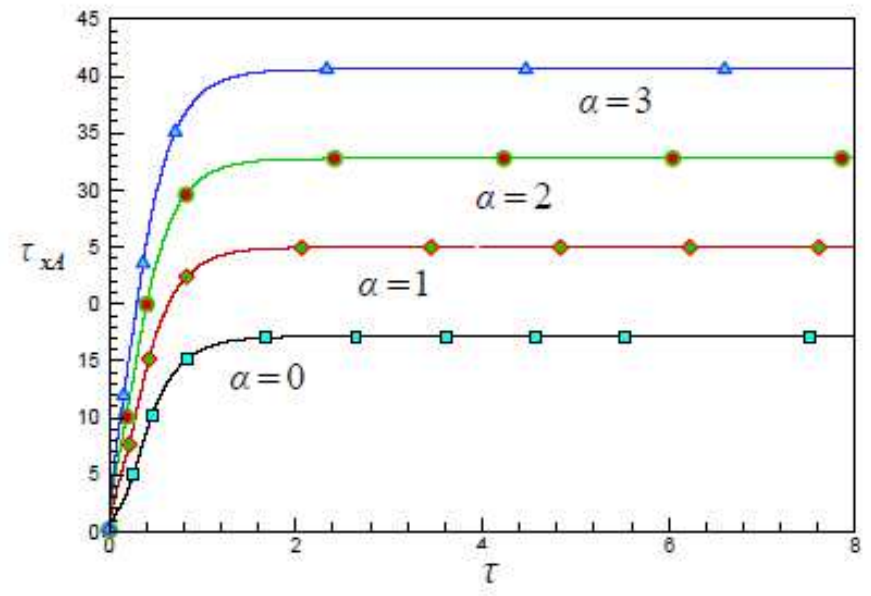

(c): Effect of $\alpha$ on $\tau_{x A}$

\section{Figure 3}

See the Manuscript Files section for the complete figure caption. 


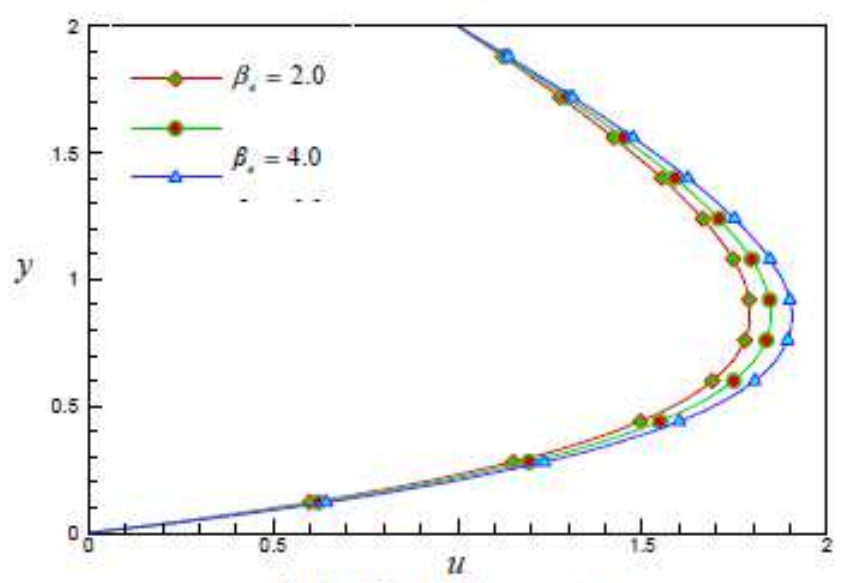

(a): Effect of $\beta_{s}$ on $u$

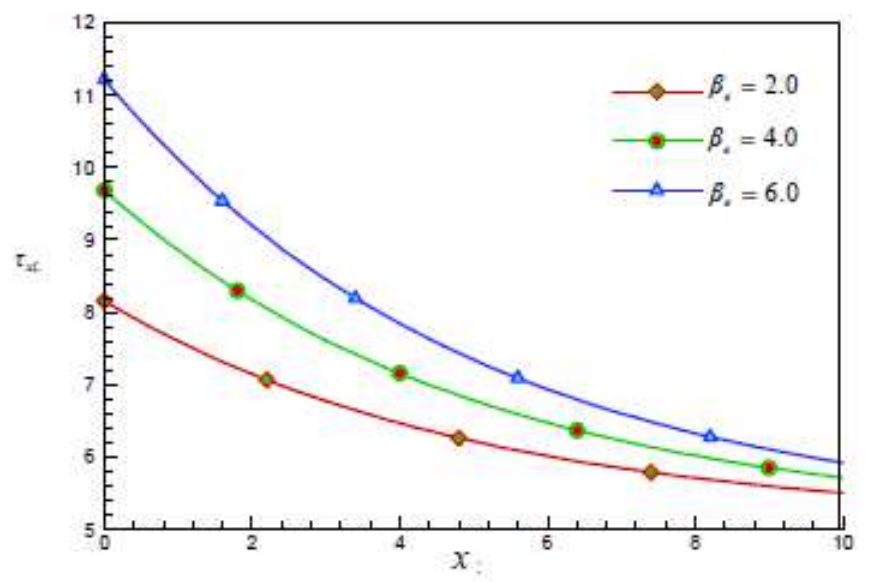

(b): Effect of $\beta_{e}$ on $\tau_{x}$

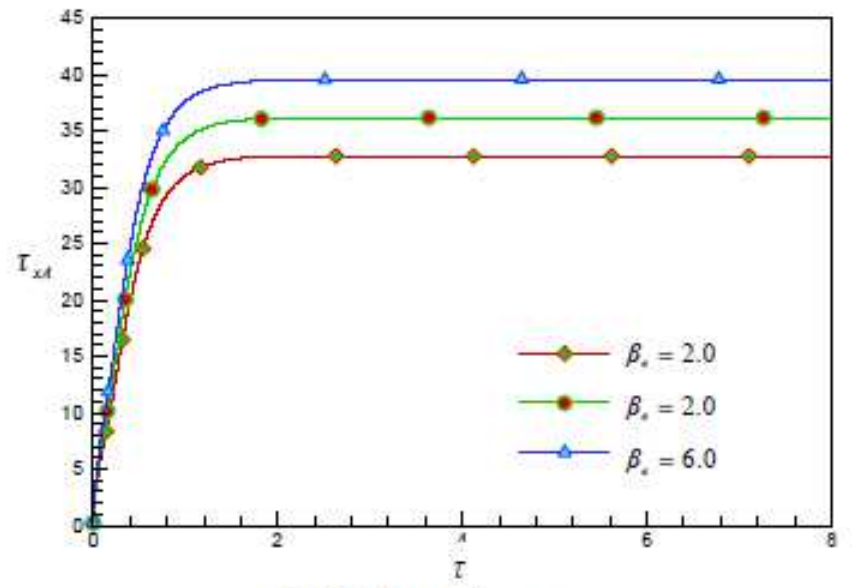

(c): Effect of $\beta_{e}$ on $\tau_{x u}$

Figure 4

See the Manuscript Files section for the complete figure caption. 


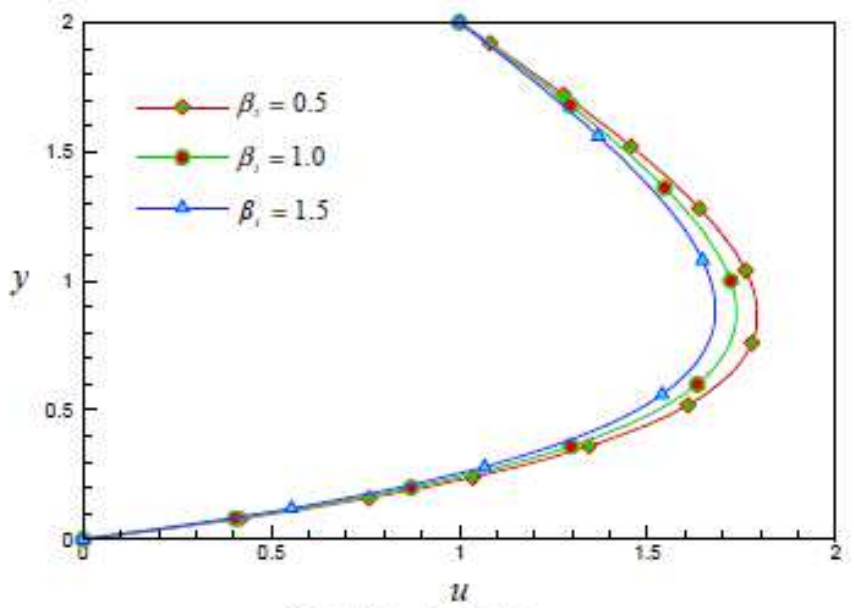

(a): Effect of $\beta_{i}$ on $u$

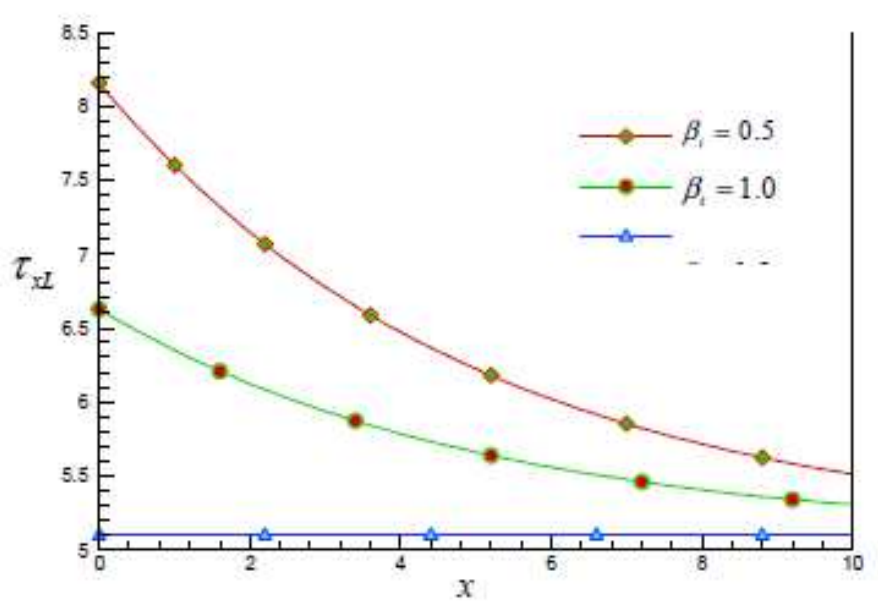

(b): Effect of $\beta_{\text {, on }} \tau_{\text {s. }}$

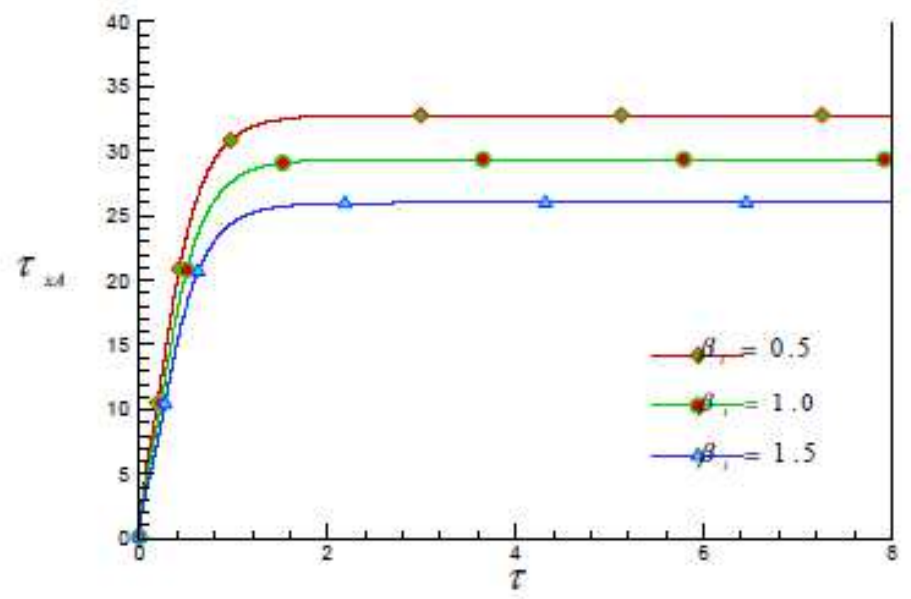

(c): Effect of $\beta_{i}$ on $\tau_{s t}$

Figure 5

See the Manuscript Files section for the complete figure caption. 


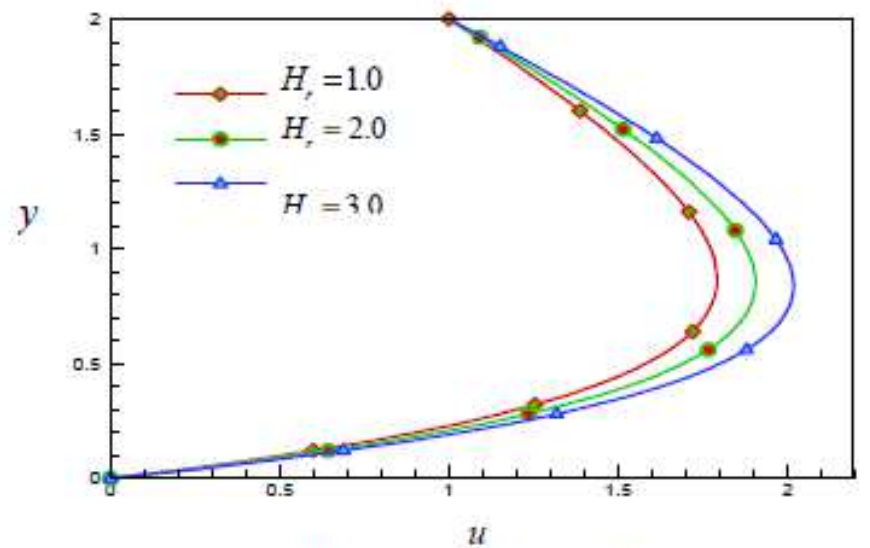

(a): Effect of $H$, on $u$

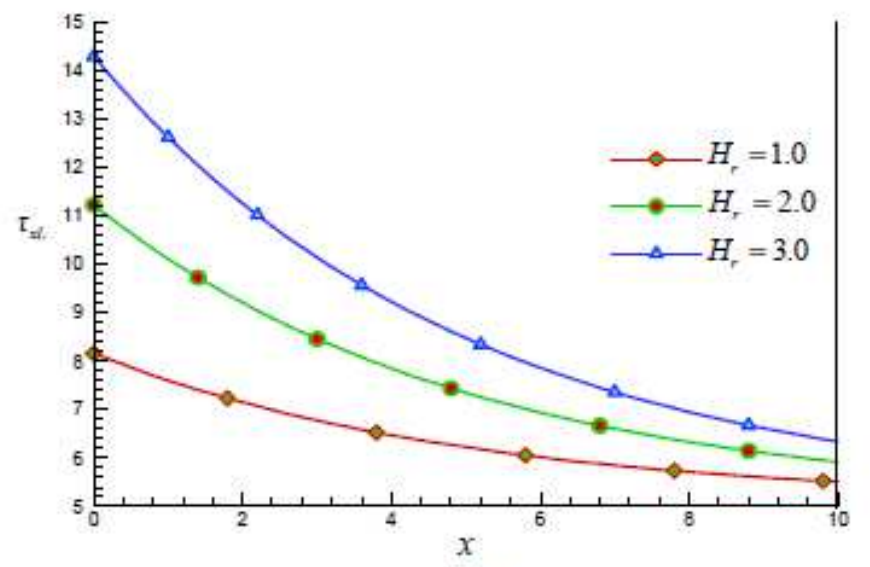

(b): Effect of $H_{r}$ on $\tau_{x I}$

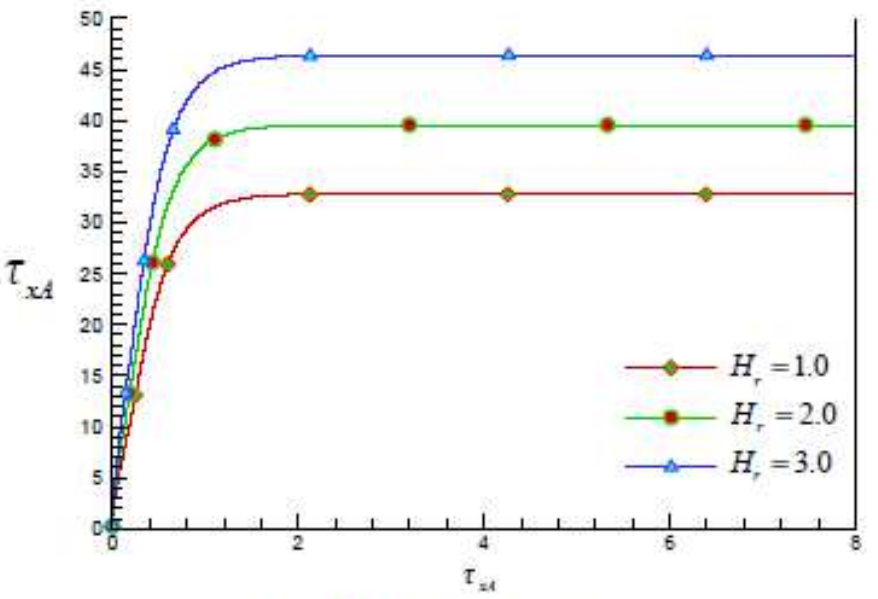

(c): Effect of $H$, on $\tau_{x i}$

\section{Figure 6}

See the Manuscript Files section for the complete figure caption. 


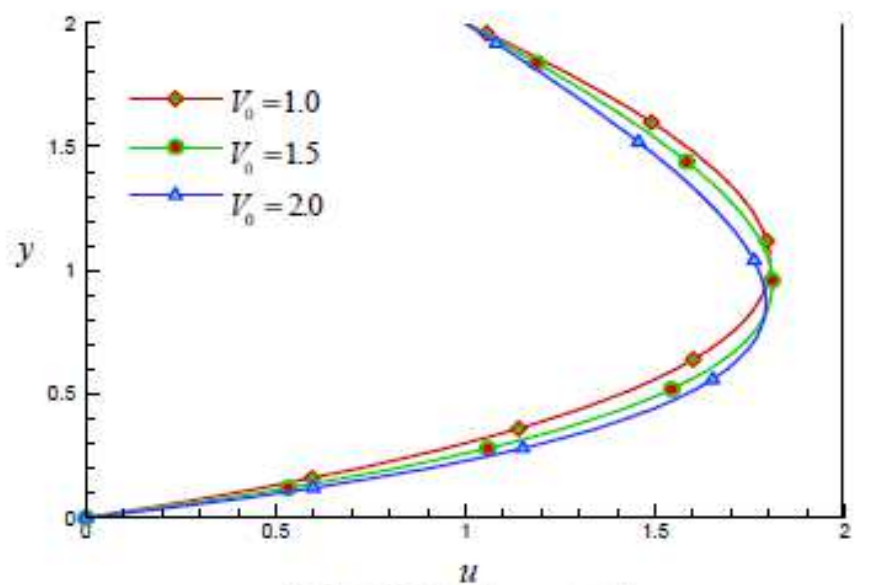

(a): Effect of $V_{0}$ on $u$

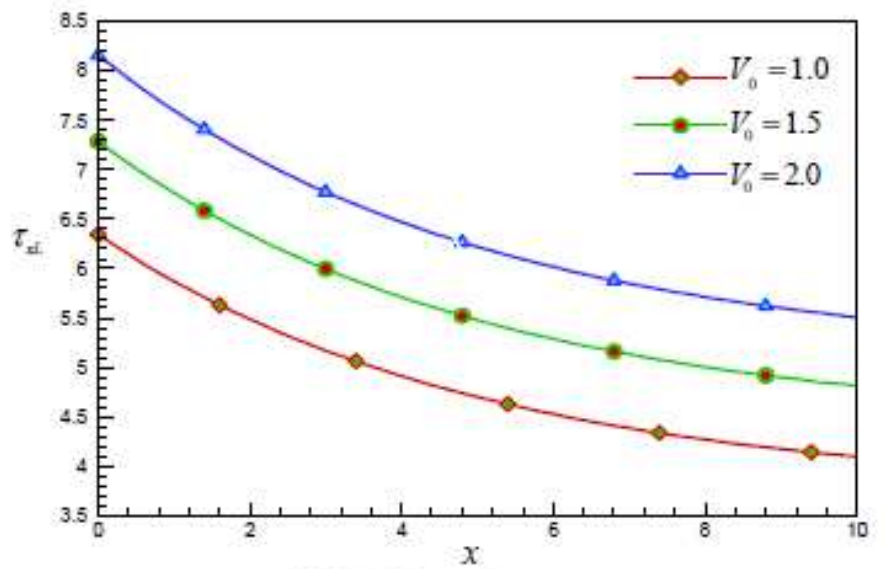

(b): Effect of $V_{0}$ on $\tau_{x I}$

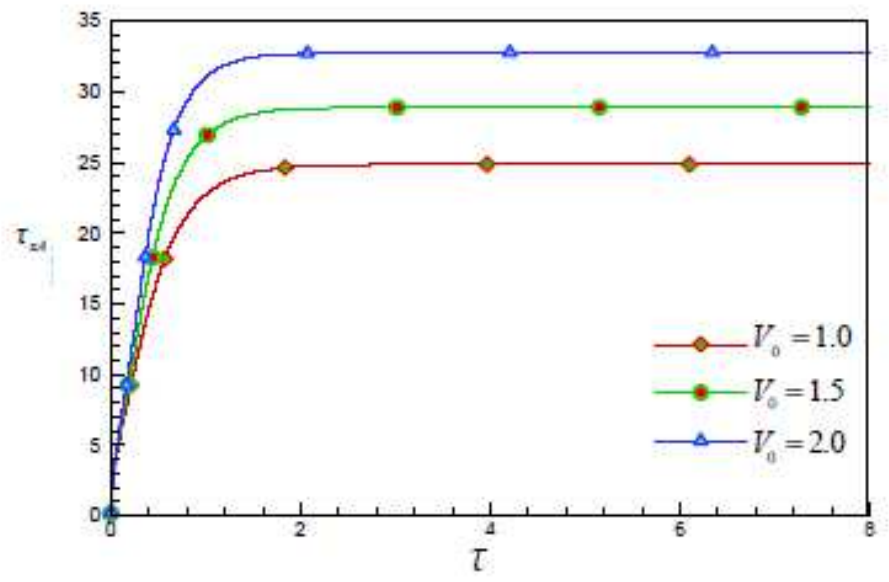

(c): Effect of $V_{0}$ on $\tau_{x i}$

Figure 7

See the Manuscript Files section for the complete figure caption. 


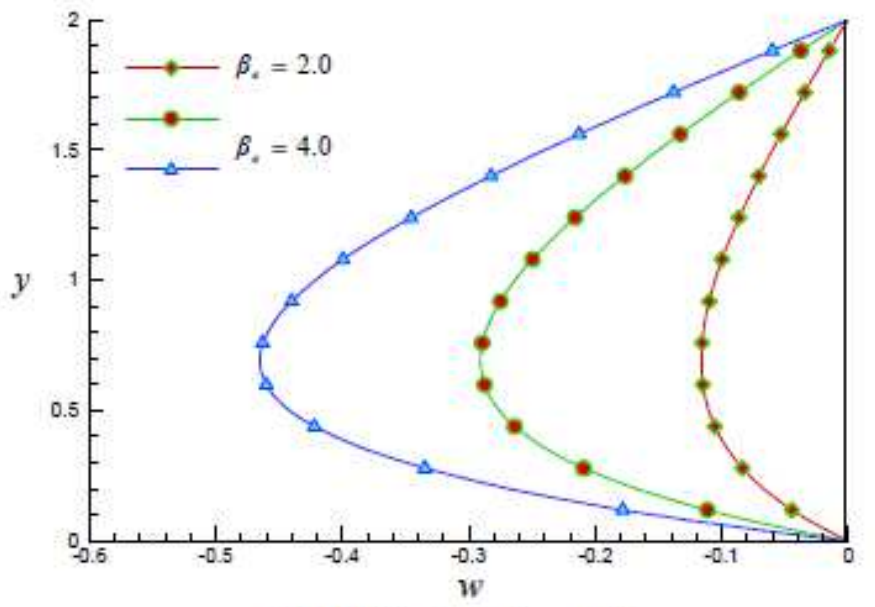

(a): Effect of $\beta_{e}$ on $w$

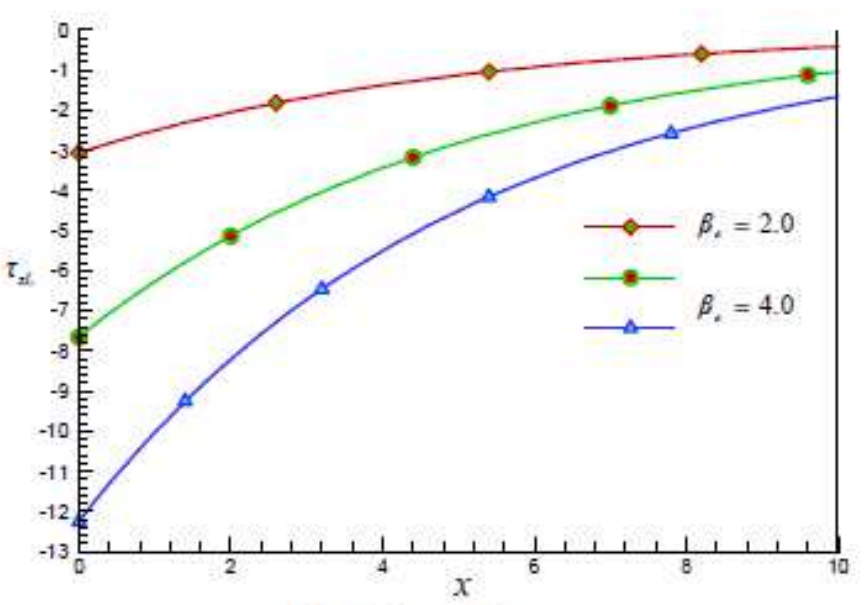

(b): Effect of $\beta$, on $\tau_{u t}$

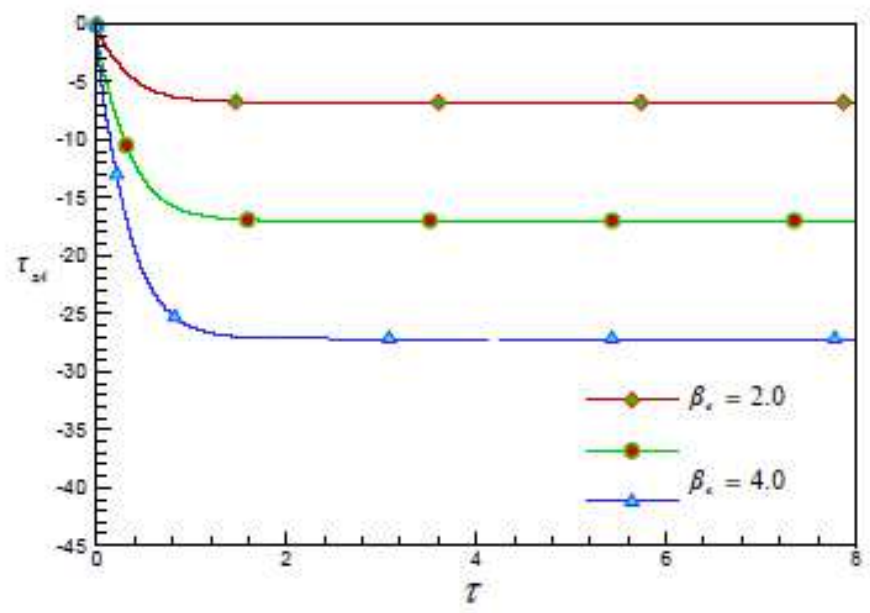

(c): Effect of $\beta$, on $\tau_{2 A}$

\section{Figure 8}

See the Manuscript Files section for the complete figure caption. 


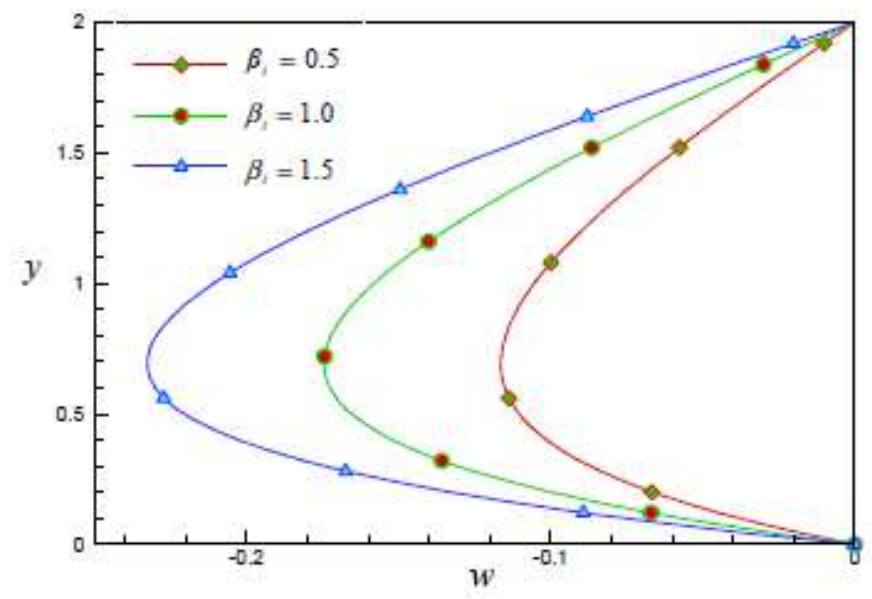

(a): Effect of $\beta_{i}$ on w

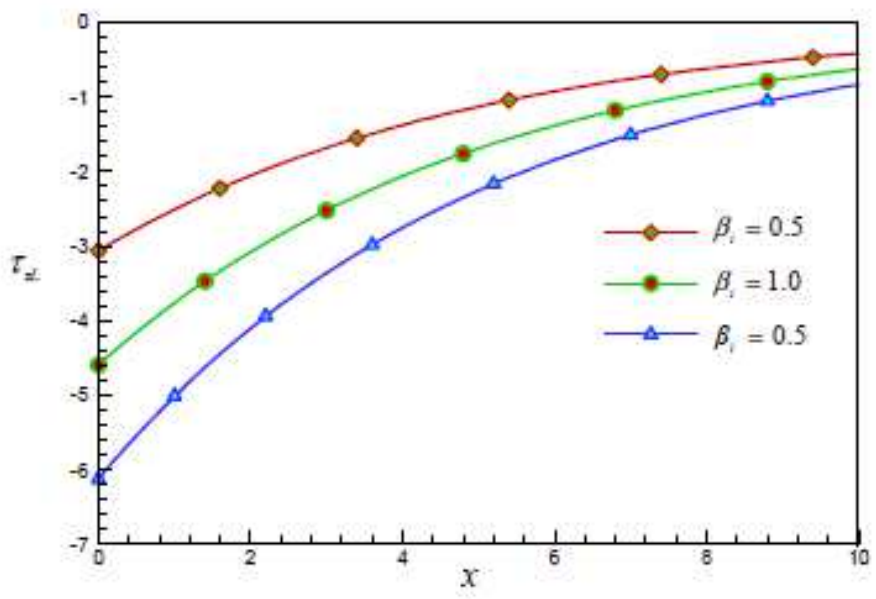

(b): Effect of $\beta$, on $\tau_{-\tau \text {. }}$

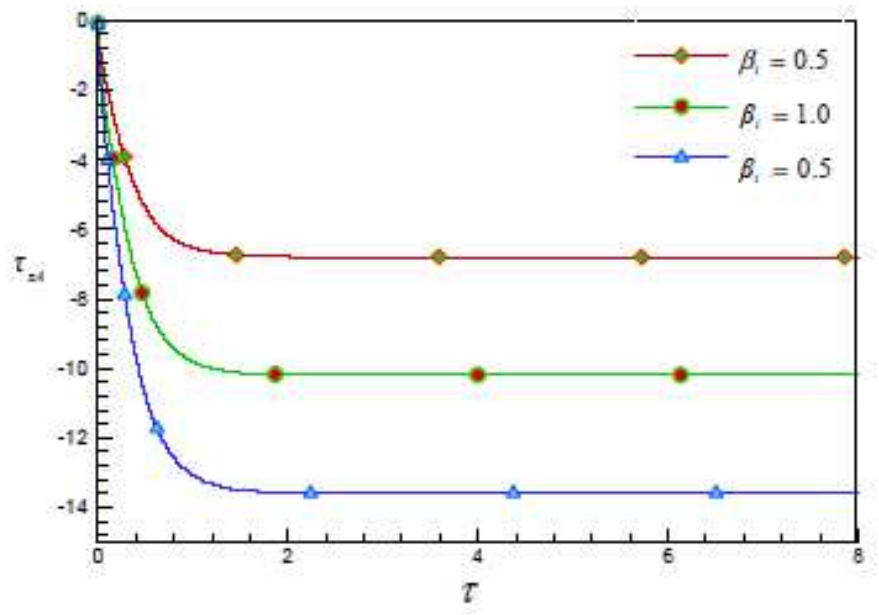

(c): Effect of $\beta$, on $\tau_{z A}$

\section{Figure 9}

See the Manuscript Files section for the complete figure caption. 


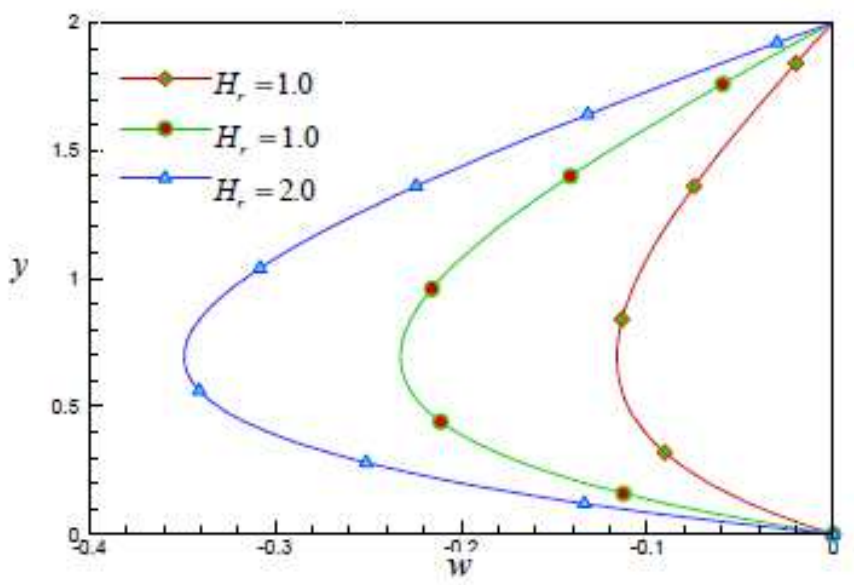

(a): Effect of $H_{r}$ on w

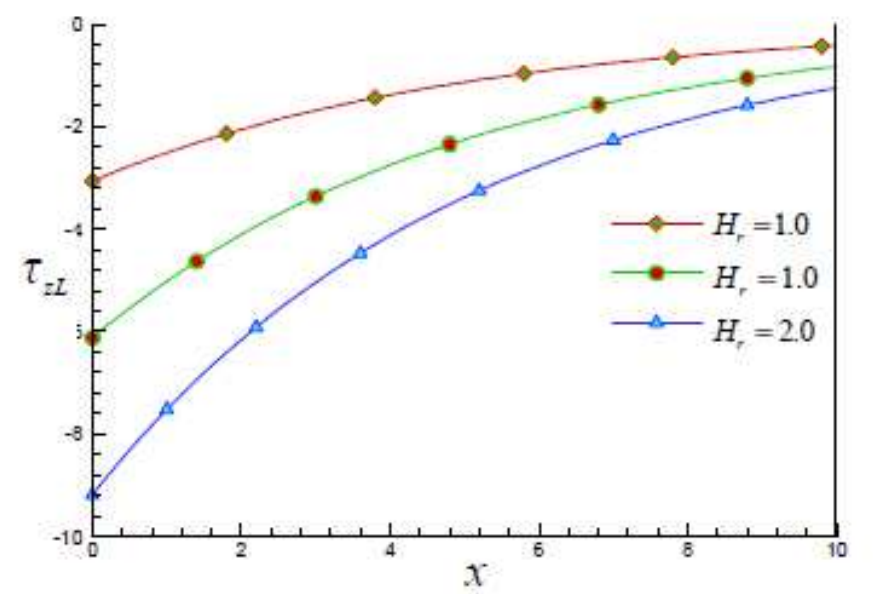

(b): Effect of $H$, on $\tau_{z L}$

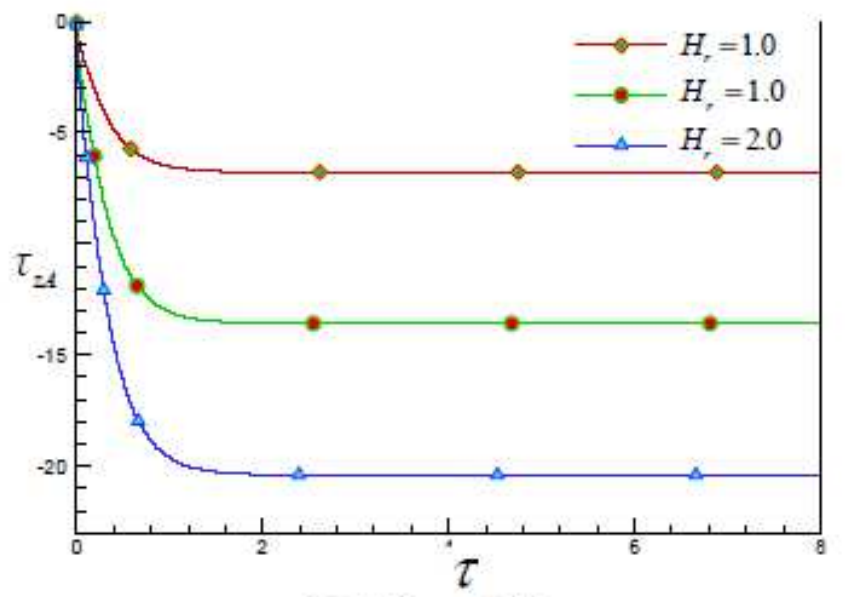

(c): Effect of $H$, on $\tau_{z A}$

Figure 10

See the Manuscript Files section for the complete figure caption. 


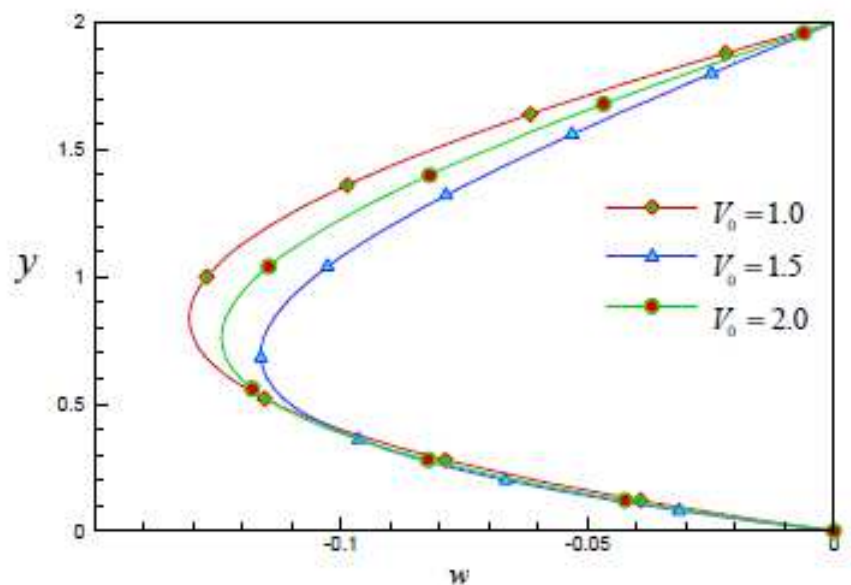

(a): Effect of $V_{0}$ on $w$

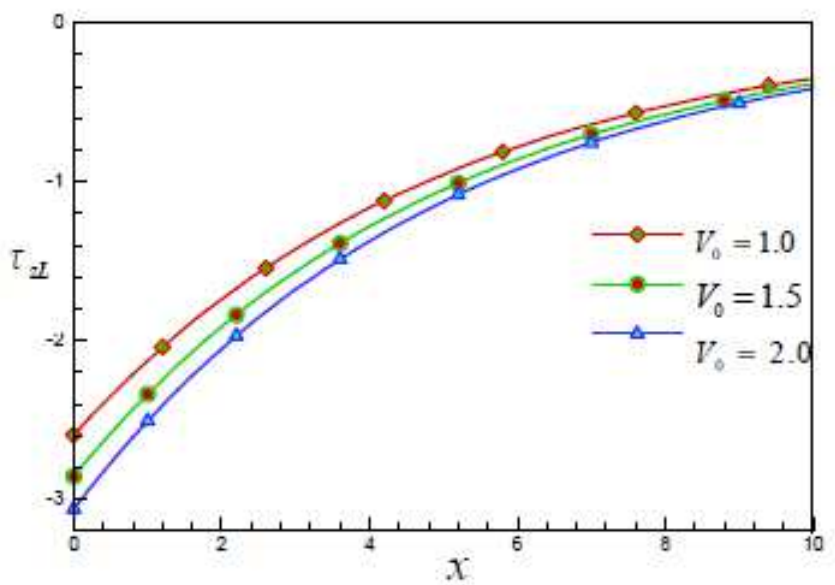

(b): Effect of $V$, on $\tau_{v I}$.

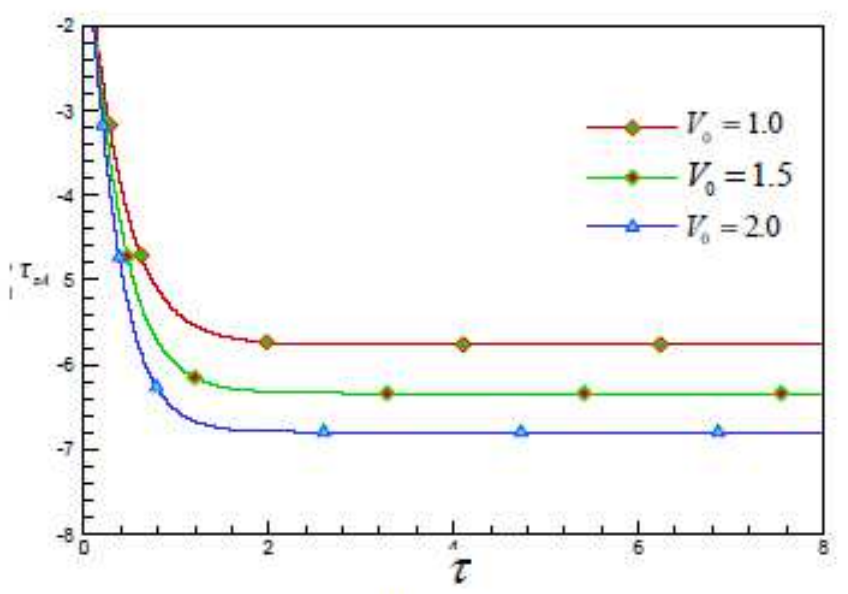

(c): Effect of $V_{0}$ on $\tau_{z A}$

\section{Figure 11}

See the Manuscript Files section for the complete figure caption. 


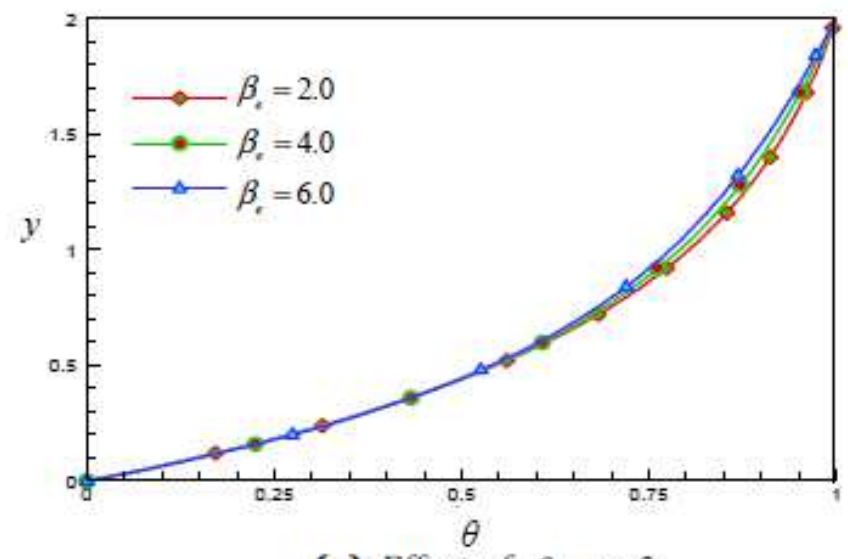

(a): Effect of $\beta_{e}$ on $\theta$

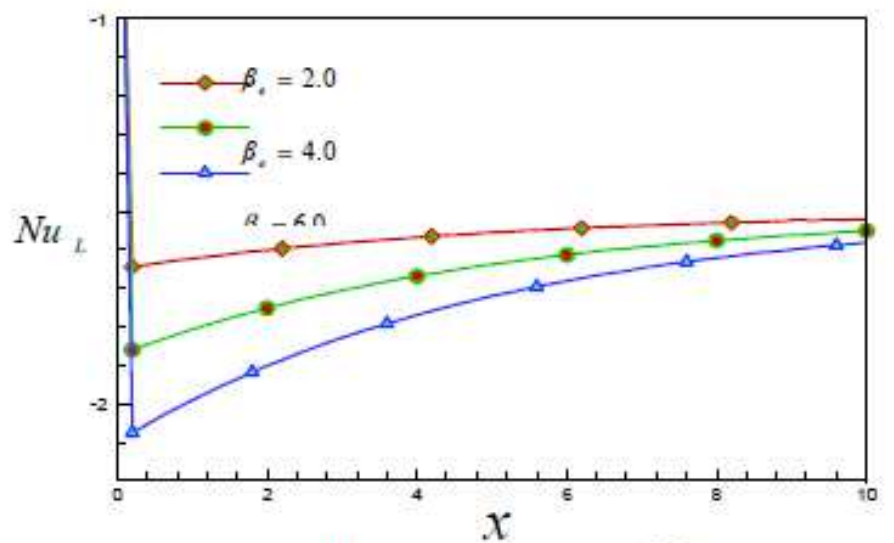

(b): Effect of $\beta$, on $N u_{I}$

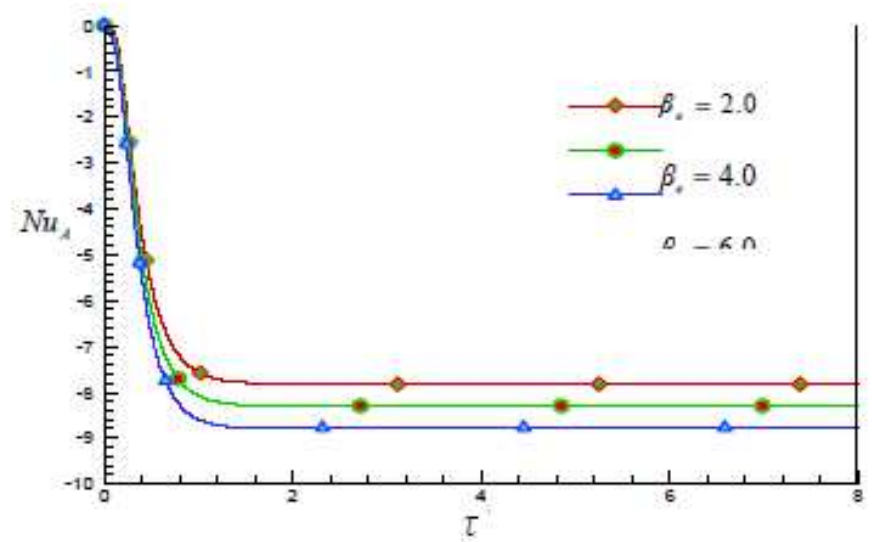

(c) : Effect of $\beta$, on $N u_{A}$

Figure 12

See the Manuscript Files section for the complete figure caption. 


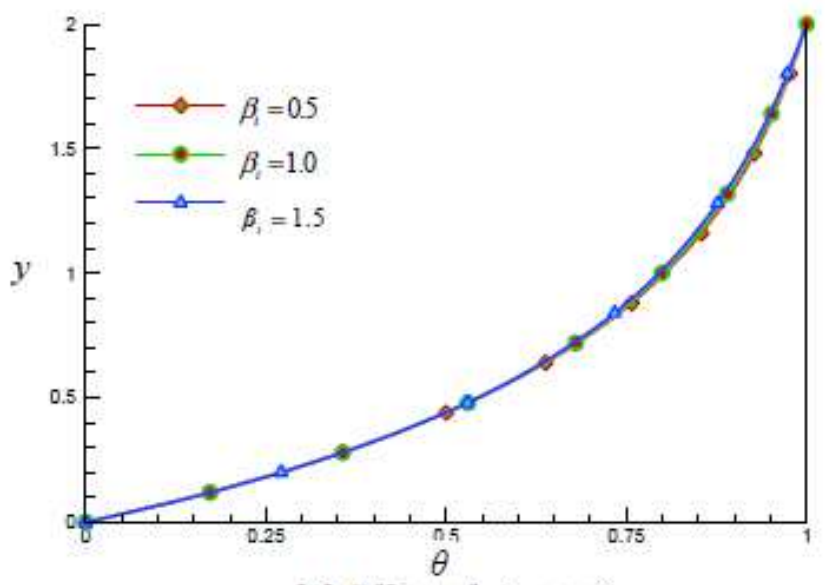

(a): Effect of $\beta$, on $\theta$

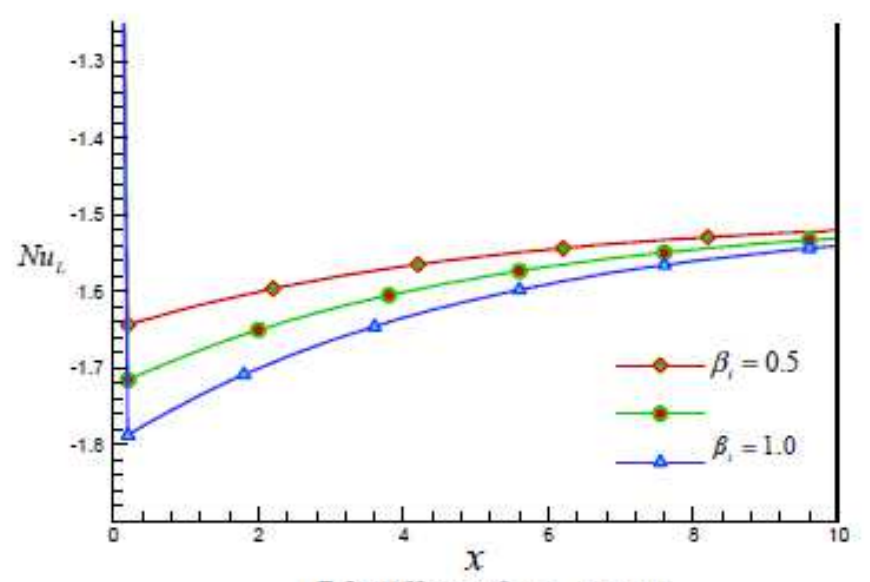

(b): Effect of $\beta$, on $N u_{L}$

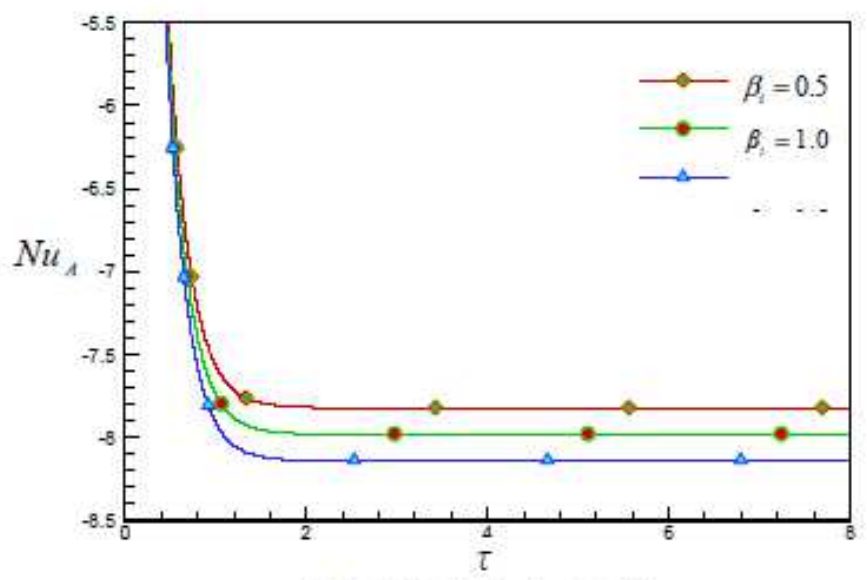

(c): Effect of $\beta_{i}$ on $N u_{A}$

Figure 13

See the Manuscript Files section for the complete figure caption. 


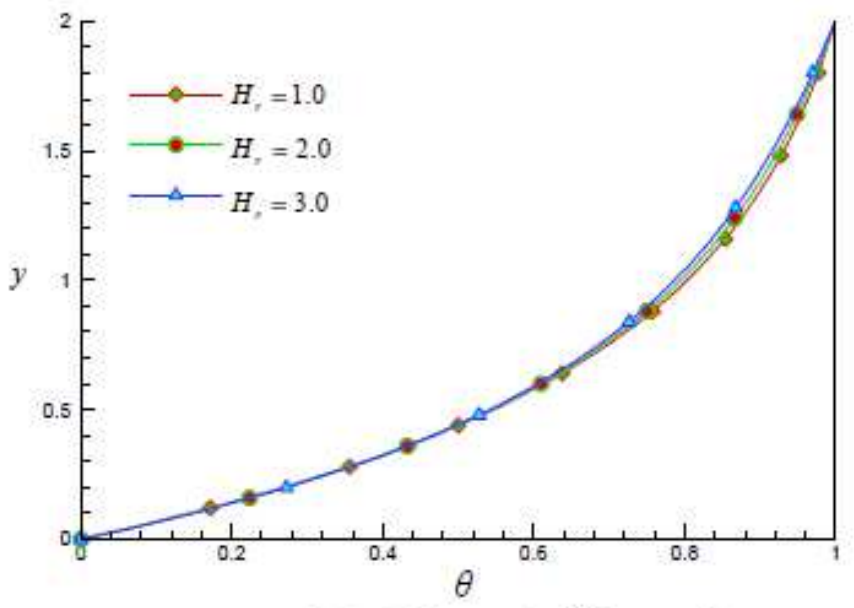

(a): Effect of $H_{r}$ on $\theta$

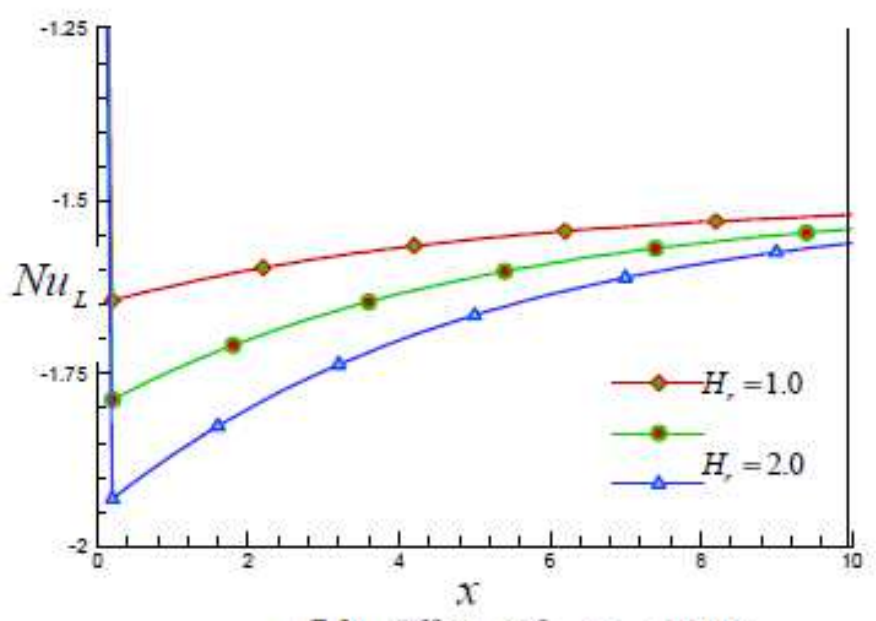

(b): Effect of $\mathrm{H}$, on $\mathrm{Nu}_{L}$

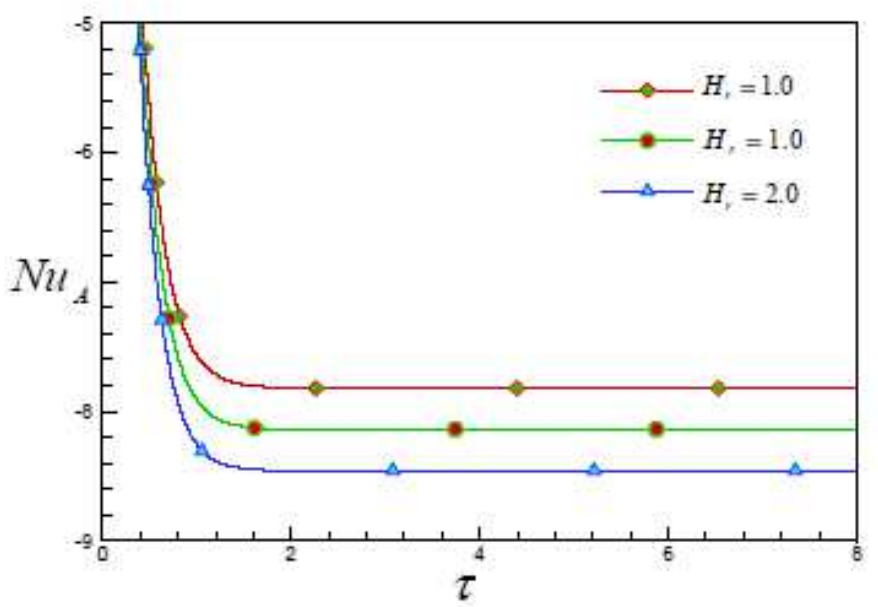

(c): Effect of $H_{r}$ on $N u_{A}$

Figure 14

See the Manuscript Files section for the complete figure caption. 


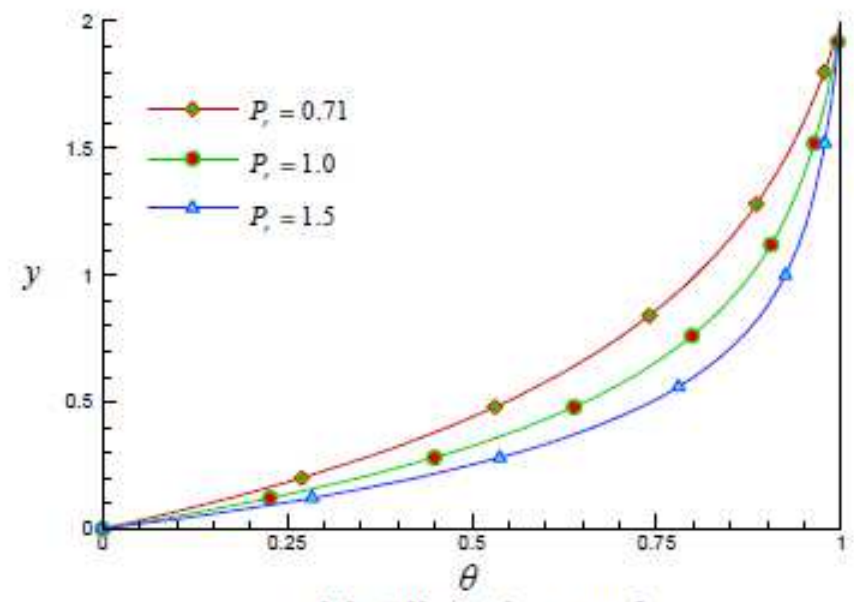

(a): Effect of $P$, on $\theta$

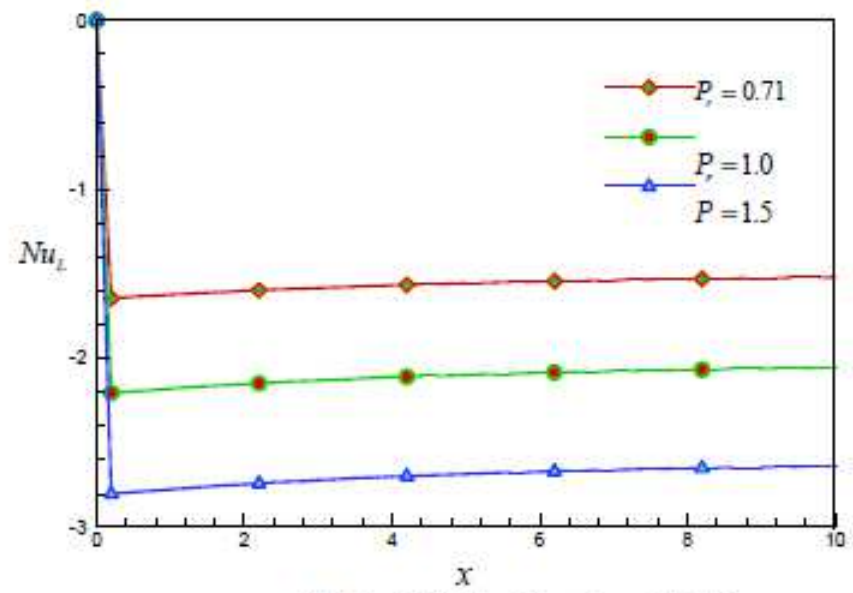

(b): Effect of $P_{r}$ on $\mathrm{Nu}_{L}$

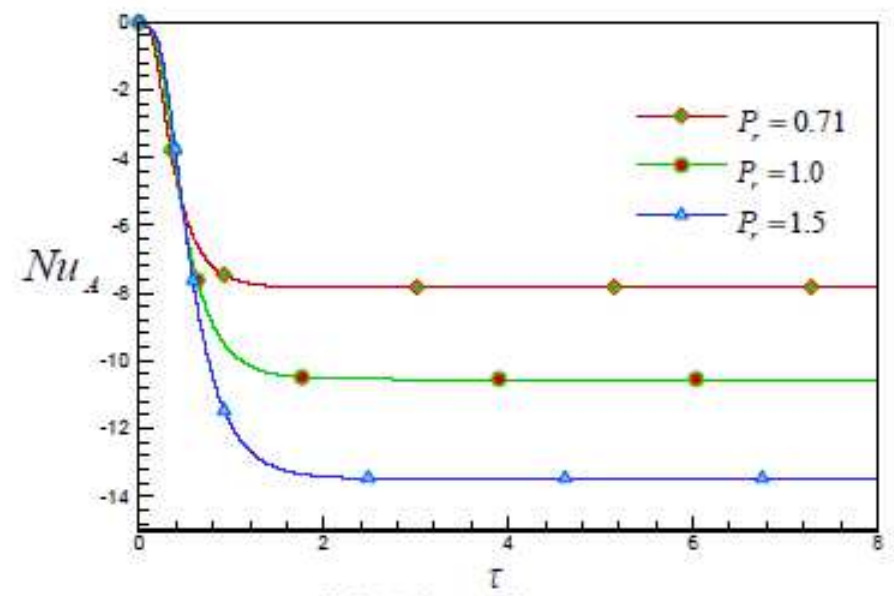

(c): Effect of $P_{r}$ on $\mathrm{Nu}_{4}$

Figure 15

See the Manuscript Files section for the complete figure caption. 


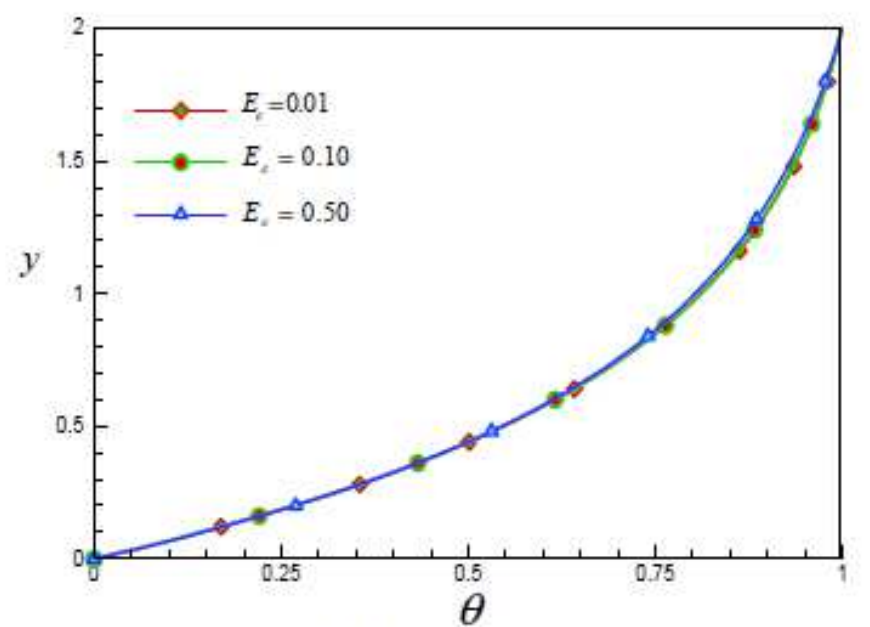

(a): Effect of $E_{c}$ on $\theta$

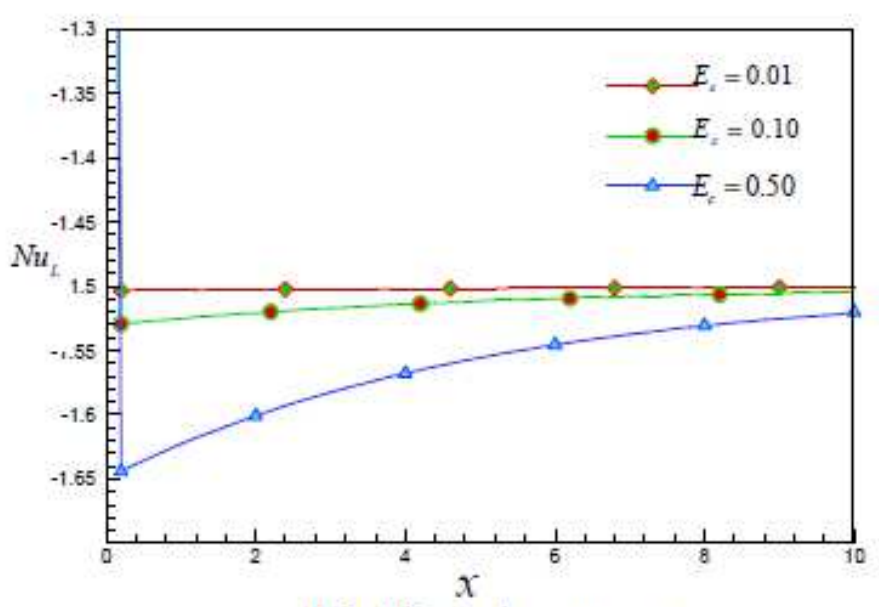

(b): Effect of $E_{c}$ on $\mathrm{Nu}_{t_{L}}$

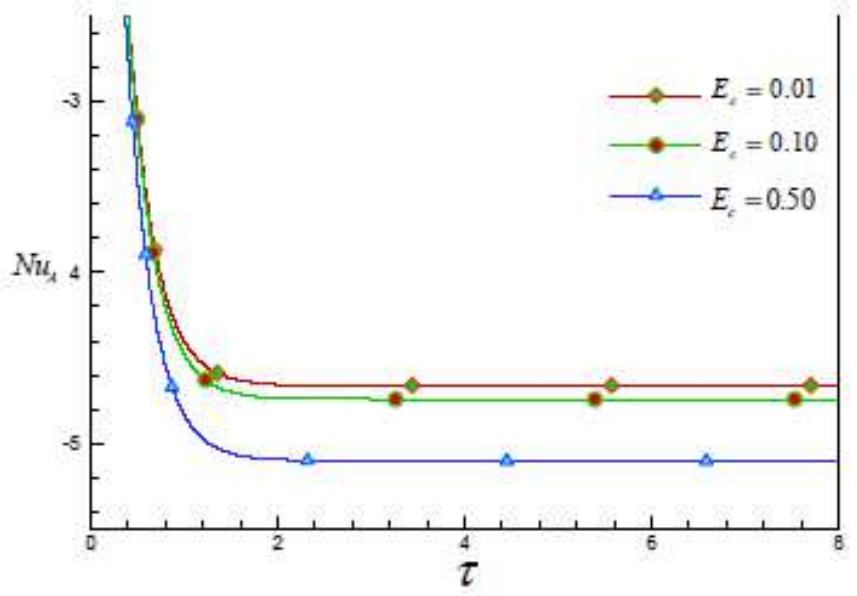

(c): Effect of $E_{c}$ on $N u_{\text {A }}$

Figure 16

See the Manuscript Files section for the complete figure caption. 


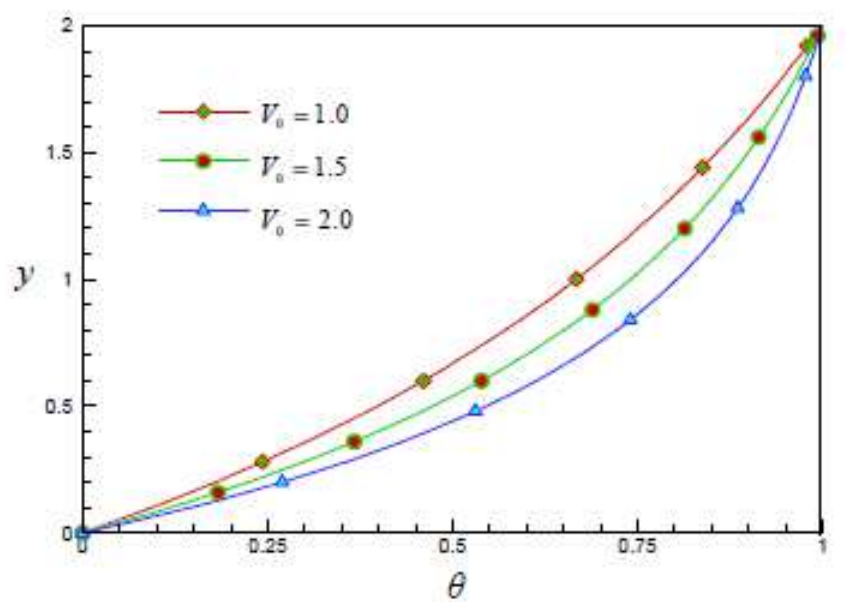

(a): Effect of $V_{0}$ on $\theta$

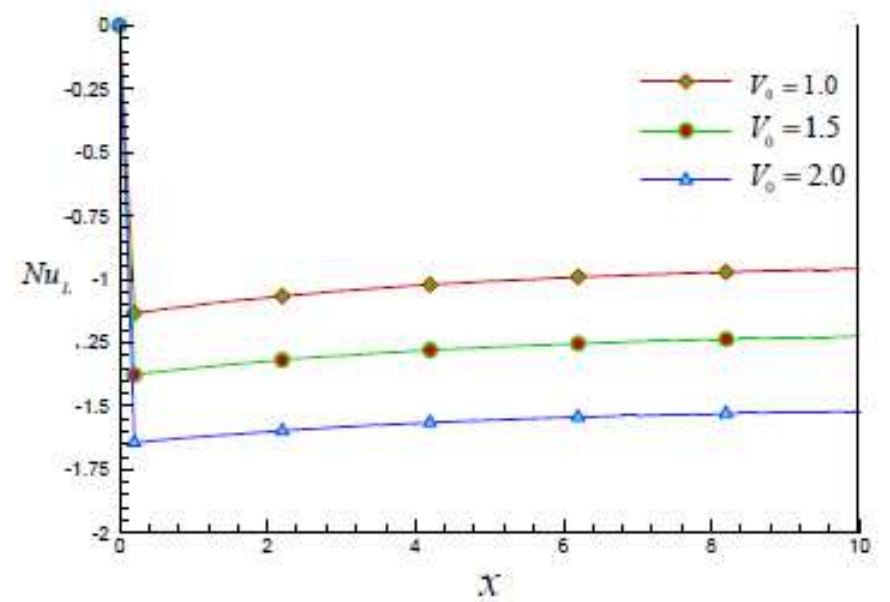

(b): Effect of $V_{0}$ on $\mathrm{Nu}_{4}$

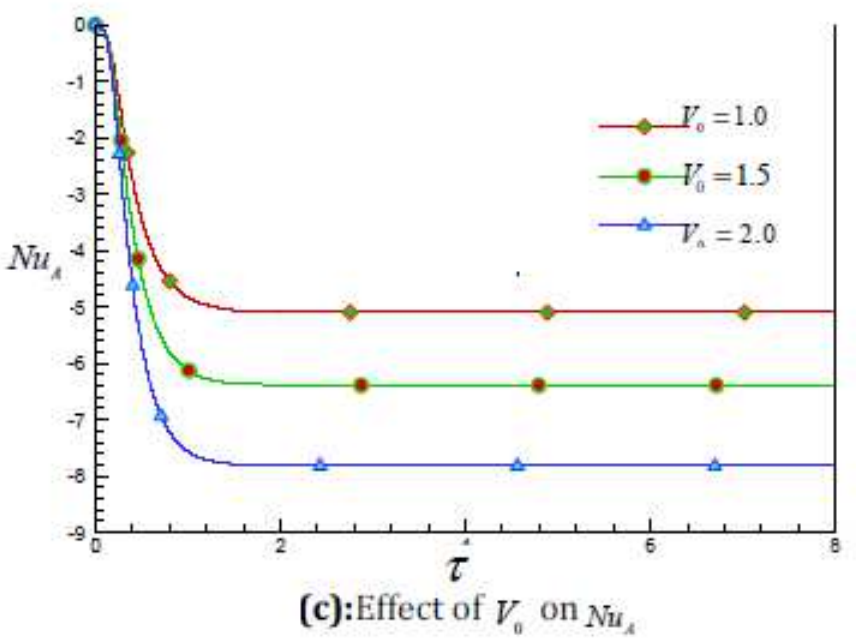

Figure 17

See the Manuscript Files section for the complete figure caption. 